\title{
Delta Oscillations Are a Robust Biomarker of Dopamine Depletion Severity and Motor Dysfunction in Awake Mice
}

\section{Timothy C. Whalen ${ }^{1}$, Amanda M. Willard ${ }^{1,2,3}$, Jonathan E. Rubin ${ }^{1,4}$, Aryn H. Gittis ${ }^{1,2 \star}$}

${ }^{1}$ Center for the Neural Basis of Cognition and

${ }^{2}$ Department of Biological Sciences and the Neuroscience Institute, Carnegie Mellon University, Pittsburgh, PA 15213

${ }^{3}$ Department of Biology and Geosciences, Clarion University, Clarion, PA 16214

${ }^{4}$ Department of Mathematics, University of Pittsburgh, Pittsburgh, PA 15260

* To whom correspondence should be addressed:

Mellon Institute, Carnegie Mellon University

4400 Fifth Ave.

Pittsburgh, PA 15213

Tel: 412-268-7229

Fax: 412-268-8423

Email: agittis@cmu.edu 


\section{Abstract}

(

Delta oscillations $(0.5-4 \mathrm{~Hz})$ are a robust but often overlooked feature of basal ganglia pathophysiology in Parkinson's disease and their relationship to parkinsonian akinesia has not been investigated. Here, we establish a novel approach to detect spike oscillations embedded in noise to provide the first study of delta oscillations in awake, dopamine depleted mice. We find that approximately half of neurons in the substantia nigra reticulata exhibit delta oscillations in dopamine depletion and that these oscillations are a strong indicator of dopamine loss and akinesia, outperforming measures such as changes in firing rate, irregularity, bursting and synchrony. We further establish that these oscillations are caused by the loss of D2 receptor activation and do not require motor cortex, contrary to previous findings in anesthetized animals.

These results give insight into how dopamine loss leads to dysfunction and suggest a reappraisal of delta oscillations as a biomarker in Parkinson's disease.

\section{Introduction}

Parkinson's disease (PD) is characterized by the loss of dopamine neurons in the substantia nigra pars compacta (SNc), inducing a state of dopamine depletion (DD) in the basal ganglia. In human PD patients, this change is accompanied by a striking increase of oscillatory power in local field potential (LFP) recordings and in the spiking of individual neurons, primarily in the beta $(13-30 \mathrm{~Hz})$ and delta/theta frequencies $(1-7 \mathrm{~Hz})$ (Lenz et al., 1988; Levy et al., 2002; Priori et al., 2004; Steigerwald et al., 2008; Du et al., 2018; Halje et al., 2019).

Of these, beta oscillations have been the primary focus of research. In PD studies, beta oscillations have been shown to correlate with symptom severity (Jenkinson \& Brown, 2011) and tend to dissipate under treatments such as dopamine replacement therapy (Weinberger et al., 2006; Ray et al., 2008). Similar oscillations are observed in some animal models of PD slightly higher in frequency $(25-35 \mathrm{~Hz})$ in 6-hydroxydopamine (6-OHDA) lesioned rats or lower (8-13 Hz) in 1-methyl-4-phenyl-1,2,3,6-tetrahydropyridine (MPTP)-treated monkeys. In these 
models, the link between beta oscillations and motor symptoms is less clear. Beta oscillations may arise later than symptoms (Mallet et al., 2008), do not consistently track symptom progression (Muralidharan et al., 2016) or reduction with treatments such as deep brain stimulation (DBS) (McConnell et al., 2012), and occur in both parkinsonian and healthy animals (Connolly et al., 2015). Attempts to artificially induce beta oscillations in these animals have also been insufficient to cause PD-like symptoms (Swan et al., 2019). Even in humans, DBS studies have shown conflicting results between the correlation of beta oscillations and motor symptoms - oscillations tend to weaken during stimulation (Kühn et al., 2006, 2008) but not in every case (Rossi et al., 2008) or may return before symptoms reemerge (Foffani et al., 2006). In contrast, the lower frequency oscillations observed in human PD patients have been much less studied, despite occurring in a greater number of neurons than beta oscillations in some patients or in the absence of beta oscillations at all (Levy et al., 2002; Du et al., 2018; Zhuang et al., 2019). These delta oscillations are often termed "tremor frequency" oscillations due to their typical coherence with Parkinsonian tremor (Bergman et al., 1994), but they may also arise without such coherence (Hurtado et al., 1999). While delta oscillations have an unclear relationship to tremor, their relationship to other PD symptoms such as bradykinesia and rigidity has not been investigated.

This lack of attention is surprising, as slower oscillations have also been observed in animal models of PD. In monkeys, oscillations as low as 3-7 Hz have been observed (Raz et al., 2000; Heimer et al., 2006; McCairn \& Turner, 2009), but these have mostly been viewed as an extension of the beta band. In anesthetized rodents, oscillations at even lower frequencies (0.5$4 \mathrm{~Hz}$ ) are most prevalent (Tseng, Kasanetz, Kargieman, Pazo, et al., 2001; Walters et al., 2007; Parr-Brownlie et al., 2009; Aristieta et al., 2016), but these have been mostly discounted as artifacts of anesthesia or artificial respiration (Ruskin et al., 2002). Indeed, delta oscillations in the striatum of 6-OHDA-lesioned rats were shown to have high coherence to anesthesiainduced slow waves in motor cortex (M1) (Tseng, Kasanetz, Kargieman, Riquelme, et al., 2001; 
Belluscio et al., 2003) and were weakened after cortical ablation (Magill et al., 2001), leading to the conclusion that they merely infiltrate the basal ganglia through $\mathrm{M} 1$ and are not relevant to the awake, behaving parkinsonian animal. Experiments investigating the presence of sub-beta band oscillations in awake, behaving animals have, to our knowledge, not been performed.

One factor limiting these investigations is the high levels of noise that contaminate low frequency signals, particularly during awake recordings. So-called "pink" or "flicker" noise is most prevalent at low frequencies and typically observed in LFP recordings but is also present in the spiking of individual neurons. This complication makes reliable detection of oscillations near or below $2 \mathrm{~Hz}$ difficult with current methods.

Here, we develop a method to reliably distinguish spike oscillations from noise and use this approach to characterize the oscillations in the substantia nigra pars reticulata (SNr) of dopamine depleted mice. We demonstrate that delta, not beta, oscillations are the primary oscillatory feature in SNr neurons after loss of dopamine, and that they correlate strongly with PD-like motor deficits. We show that, contrary to prior reports, delta oscillations in the SNr precede those in M1, and that M1 is not necessary for these oscillations to develop in the SNr. We also establish that a loss of D2 receptor activation is sufficient to immediately and reversibly generate both delta oscillations and PD-like akinesia in awake mice, suggesting a direct link between dopamine loss, delta oscillations, and parkinsonian symptoms. This work indicates that delta oscillations in basal ganglia neurons are a critical component of parkinsonian pathology in DD mice and suggests that DD mice may effectively model the low frequency oscillations seen

73 in PD patients.

\section{Results}

\section{Dopamine depleted mice exhibit $0.5-4 \mathrm{~Hz}$ spike oscillations in SNr units}

We recorded single units from the substantia nigra pars reticulata (SNr) of awake, head- 
investigate oscillations in the spiking activity of single units, we first examined spike trains and their autocorrelograms. In control animals, units typically fired in a regular, pacemaking pattern, indicated by a fast oscillation in their autocorrelograms which corresponded to the interspike interval of pacemaking and flattened within 20-100 ms (Figure 1c). In contrast, units in bilaterally dopamine depleted animals exhibited autocorrelograms that showed much slower oscillations between 0.5 and $4 \mathrm{~Hz}$ that remained autocorrelated for several seconds, visible in the raw spike trains as peaks and troughs or pauses in firing (Figure 1d). These slow oscillations were never observed in the autocorrelograms of units from control animals.

\section{Phase shift analysis enables distinction between low frequency oscillations and neural noise}

We first sought to reliably quantify these oscillations in dopamine depleted mice. Neural noise is more prevalent in awake than anesthetized animals, and typically manifests in a power law fashion (called "pink" or "flicker" noise) such that it is dominant in low frequencies. Since the oscillations we observe in SNr units in DD were in the range typically tainted by pink noise, we could not reliably detect them using standard approaches based solely on the power spectral density or transformations of it. Specifically, random peaks in the power spectral density atop pink noise, or the pink noise itself, can easily be misidentified as an oscillation of interest (Figure 2c).

To overcome false positive detections, we used both the power and phase information provided by the short time Fourier transform to identify oscillatory components of spike trains with consistent phase over time (see Methods). By requiring that an oscillation have both high spectral power and low phase shift (Figure 2a), we successfully distinguished the oscillations of interest embedded in pink noise from the noise itself (Figure 2b). Notably, spike trains that exhibit a relatively flat autocorrelation but have delta peaks in their PSD are successfully disregarded as oscillators when phase shift analysis is applied (Figure 2c). 


\section{Delta, not beta, oscillations in SNr units are a marker of dopamine depletion}

Using this detection method, we observed that very few SNr units from control animals exhibit an oscillation in the $0.5-4 \mathrm{~Hz}$ range (2 of 85 units pooled across animals), whereas in each bilaterally dopamine depleted animal, 33-92\% of units exhibited significant delta oscillations (117 of 226 units pooled) three days after depletion (Figure 3a). Without using the phase shift criterion, a much greater number of units were flagged as oscillating, particularly in control mice ( $28 \%$ of units vs $2 \%$ after phase shift correction, Figure $3 b$ ), despite these units having a nearly flat autocorrelation as in Figure 2c. To determine whether these oscillations remained stable at longer time points after depletion, we recorded from the SNr of unilaterally depleted mice, 2-4 weeks after depletion. We found that a significant proportion of SNr neurons still exhibited delta oscillations at these later time points (22-82\% for each animal, 48 of 83 units pooled), suggesting that these oscillations are a stable feature of basal ganglia pathophysiology following dopamine depletion. A small number of units on the contralateral side of the lesion also exhibited delta oscillations (0-19\% for each animal, 7 of 72 units pooled) (Supplemental Figure 1).

To ensure that delta oscillations were not merely an immune or inflammatory side effect of the injected toxin or cell death, we treated a cohort of animals intraperitoneally with reserpine, a compound that blocks the vesicular monoamine transporter 2 (VMAT2) complex from packaging monoamines into vesicles. This yielded a monoamine (including dopamine) depletion without any intracranial injection or cellular death and produced akinetic symptoms similar to those observed in bilateral 6-OHDA depleted mice. When we recorded three days after the start of daily reserpine injections, these animals exhibited a high proportion of slowly oscillating units in the SNr (33-100\% for each animal, 74 of 119 units pooled), similar to bilaterally depleted animals (Figure 3a).

Given the prevalence of beta oscillations in the DD and PD literature, we sought to determine if these animals' SNr units also exhibited beta oscillations. We defined a wide 
131 frequency range for beta oscillations, 7-35 Hz, which fully encompasses the definition of beta

132 oscillations across humans and common model species (monkey and rat). We saw no increase

133 in the fraction of beta oscillating units after any form of dopamine depletion, with or without our

134 phase shift criterion (Figure $3 c-d$ ). Taken together, our results suggest that delta, not beta,

135 oscillations are the primary oscillatory feature in SNr spike trains of awake, dopamine depleted

136 mice.

Oscillations predict DD severity and behavior better than other physiological measures

\section{of dysfunction}

To understand how delta oscillations relate to the severity of dopamine depletion, we used an existing dataset of SNr recordings from mice gradually depleted to varying levels of dopamine loss through successive small injections of 6-OHDA (Willard et al., 2019). In this data, we looked at the relationship between an animal's fraction of units exhibiting a delta oscillation and its level of dopamine neuron loss (as measured by striatal tyrosine-hydroxylase (TH) immunoreactivity). Performing a linear regression to predict \% $\mathrm{TH}$ remaining from oscillation fraction showed a relatively strong $\left(r^{2}=0.5267\right)$ and significant $(p<0.01$ from a bootstrapped $99 \%$ confidence interval, see Methods) relationship between dopamine loss and the fraction of oscillating units (Figure 4a).

Since striatal TH immunoreactivity is not a perfect indicator of parkinsonian symptoms, we also used these measures to predict motor behavior. Prior to in vivo recordings, these

151 animals were given a series of behavioral tests to measure their mobility, dexterity, and strength

152 (see Methods \& Willard et al., 2019), and we performed principal component analysis on the 153 results of these tests to get a single measure - the first principal component (PC1) - of their 154 motor deficits. A linear regression predicting PC1 from the fraction of oscillating units illustrated 155 a similarly strong and significant relationship (Figure $4 b, r^{2}=0.6406, p<0.01$ ). 
Besides oscillations, many other neural measures in the basal ganglia have been

suggested as correlates of DD severity - most commonly, changes in firing rate, firing regularity, burstiness, and synchrony between units. To see how delta oscillations compare to these measures in reliably predicting DD severity, we built a set of statistical models to predict \% TH in each animal from five physiological parameters measured from single units in the SNr: 1) median firing rate, 2) median coefficient of variation (CV) of interspike intervals (ISIs), 3) median rate of bursts, as measured from the Poisson surprise test, 4) fraction of significantly synchronous pairs of units, and 5) fraction of units with significant $0.5-4 \mathrm{~Hz}$ oscillations. Due to the highly nonlinear relationship between the first four of these measures and DD severity (Willard et al., 2019), we performed a series of nonlinear regressions on this data by building 1000 decision trees from randomly selected sets of 20 (out of 25) animals, excluding the remaining 5 animals as a testing set for each tree (Figure 4c). We estimated a 95\% confidence interval of mean squared errors (MSE's) from these 1000 trees and showed that a tree built from these parameters predicts $\mathrm{TH}$ significantly better than a naive intercept-only model (Figure 4d).

To determine how each parameter informs the model, we shuffled the testing data for that parameter and calculated how much this loss of information increased the MSE of the model (the 'importance" of that parameter). We then estimated 95\% confidence intervals for the importance of each parameter (see Methods). The fraction of oscillating units was the only parameter whose confidence interval did not extend below zero (Figure 4e), suggesting that, when the model is built to include oscillations, they are the only parameter that provides reliably predictive information. In other words, while other parameters may provide information, that information is redundant when the fraction of oscillating units is known.

To confirm this in another manner, we rebuilt the models using the same cross-validated training and testing sets as above using only a single parameter at a time, or using all of the parameters except oscillations. The first four parameters fall outside (FR) or on the edge (CV 
182 ISI, Burst and Sync) of the full model confidence interval, but the model with all four parameters

183 performs better than any individual parameter (Figure 4d), confirming results seen previously

184 (Willard et al., 2019). However, the model built using only oscillations as a predictor is, on

average, better than any other model including the combined parameter model, providing further evidence that other physiological parameters are not additionally informative when oscillations are considered.

Using the same procedure as above to predict PC1 of the animals' behavior, we found very similar results to those predicting $\mathrm{TH}$ levels - namely, firing rate, irregularity, burstiness and synchrony provide some information in predicting behavior, particularly when considered together. However, when the fraction of oscillatory units is included in the model, it is the only important variable, and is significantly so, in predicting motor dysfunction (Figure $4 \mathrm{f}-\mathrm{g}$ ).

\section{Delta oscillations arise immediately following loss of MFB transmission or D2 receptor}

\section{activation}

The mechanism behind the observed delta oscillations is unclear, but they could arise within 2 minutes of the start of lidocaine infusion (before infusion ended) and waned within ten minutes after the end of infusion, mirroring the time course of akinesia observed during the experiment (Figure 5a-c). This result is consistent with the similarly rapid onset of slow oscillations produced by TTX infusion to the MFB under anesthesia (Galati et al., 2010) and

206 demonstrates that low frequency oscillations arise in the SNr almost immediately after loss of

207 MFB transmission, ruling out long-term mechanisms for their generation. 
To determine whether the loss of dopamine signaling is causal to the onset of delta oscillations, we recorded from the SNr of healthy animals before and during the systemic injection of a D1-receptor (D1R) antagonist (SCH233890) or a D2-receptor (D2R) antagonist

211 (raclopride). While both drugs caused reduced movement on the wheel, only the D2R

212 antagonist led to the development of oscillations in the SNr (Figure $5 d-f$ ). We then performed

213 the converse experiment, injecting a D1R agonist (SKF81297) or D2R agonist (quinpirole)

214 systemically into bilateral DD animals. Similarly, while both led to highly increased motor activity

215 (though highly dyskinetic in the case of D1 agonism), only the D2R agonist injection attenuated delta oscillations in the SNr (Figure $5 \mathrm{~g}-\mathrm{i})$. This suggests that low frequency oscillations are mediated purely due to a loss of action on D2Rs and are not affected by D1Rs.

Delta oscillations are a feature of dopamine depletion throughout the indirect pathway

222 thus recorded from healthy and dopamine depleted globus pallidus externa (GPe) (Figure 6a)

223 and subthalamic nucleus (STN) (Figure 6c), two reciprocally connected nuclei in the indirect

224 pathway that both project heavily to SNr. We found a similar pattern of oscillatory activity across 225 units in the GPe (40-80\% of units in each animal, Figure $6 \mathrm{~b})$ and STN (15-70\% of units in each 226 animal, Figure 6d) after dopamine depletion, whereas only 1 of 111 total GPe and 1 of 63 STN 227 units exhibited oscillations in the healthy state.

\section{Two populations of delta oscillating units in SNr both lead oscillations in motor cortex}

Previous literature suggests that oscillations in the dopamine depleted basal ganglia

231 arise due to input from oscillating neurons in motor cortex (M1) under anesthesia (Tseng,

232 Kasanetz, Kargieman, Riquelme, et al., 2001). However, since we have shown that these 233 oscillations arise from antagonism on D2R's, a receptor more prevalent in the basal ganglia 
than $\mathrm{M1}$, a possible alternative in awake animals is that these oscillations arise first in the basal ganglia and then entrain M1.

To distinguish between these possibilities, we sought to characterize oscillations in the M1 of DD animals and determine the phase lag between M1 and SNr oscillations. We recorded an electrocorticogram (ECoG) in $\mathrm{M} 1$ while simultaneously recording from single units in $\mathrm{SNr}$ (Figure 7a). Compared to healthy controls, the M1 ECoG of DD animals exhibited a large increase in delta oscillations and reduction in theta $(4-7 \mathrm{~Hz})$ oscillations, which are typically seen in the cortex of healthy mice (Tort et al., 2018) (Figure 7b-c).

Determining the relationship between two oscillating signals from their phases is a difficult task - if the phase of one perfect oscillator slightly leads that of a second perfect oscillator, it is impossible to distinguish whether the first leads the second at a short lag or if the second leads the first at a long lag. However, neural oscillations do not match the activity patterns of perfect oscillators, but in fact have profiles that vary across periods and highly varying period lengths that are merely centered on a range of values. We can leverage this fact to make predictions about the relative timing of SNr and M1 (Figure 7d).

To quantify this relation, we performed a series of Granger causality regressions, which make no assumptions on the periodicity of the signals. Rather, they simply attempt to predict changes in M1 ECoG based on its own history (the null, autoregressive model) or by additionally including SNr spiking information from a single unit. For each unit, we computed 201 separate models predicting M1, each using SNr spiking information at a different lag between -1 (i.e., past spikes) and +1 seconds (i.e., future spikes). Aligning the lag coefficients of the models for a single unit illustrates a periodicity in their values that matches the oscillation period (Figure 7e-f).

We computed the mean squared error (MSE) of each model at each lag and considered the lag that minimized MSE. To quantify whether this model significantly outperforms the purely autoregressive ECoG model, we performed an F test on the two models, correcting for multiple 
260

261

262

263

264

265

266

267

268

269

270

271

272

273

274

275

276

277

278

279

280

lag comparisons (Figure 7e-f). We find that 51 of 63 of oscillating units in SNr predicted changes in the ECoG significantly better than the null autoregressive model, suggesting that there is significant correlation between $\mathrm{SNr}$ and $\mathrm{M} 1$ at a consistent time lag.

When analyzing the regression coefficients at these significant lags, we found a clear bimodal distribution of units determined by whether the active or inactive phase of their spike oscillation predicted positive deflections in M1. We term these types "active-predicting" (AP) units, which make up approximately $47.1 \%$ of ECoG-locked units $(38.1 \%$ of oscillating units, $34.3 \%$ of all analyzed units) and "inactive-predicting" (IP) units (Figure $7 \mathrm{~g}$ ), which make up the remaining $52.9 \%$ of ECoG-locked units (42.9\% of oscillating units, $38.6 \%$ of all analyzed units). We see further evidence of these two distinct populations through cross correlation analysis of SNr unit pairs (Supplementary Figure 2).

When clustering units based on their phase lag relative to $\mathrm{M} 1, \mathrm{SNr}$ units also organize into a bimodal distribution, with one mode dominated by AP units and the other by IP units.

(Figure 7h). Critically, all significant lags were negative - that is, SNr spikes from both populations of SNr units consistently predicted future changes in the ECoG, but not the inverse (Figure 7h). The relative timings of these signals suggest an order in which oscillations propagate through the SNr and cortex - AP neurons enter their active phase (increase firing), then IP neurons enter their inactive phase (decrease firing or pause), and finally M1 enters its active phase. These results suggest a consistent timeline of oscillatory dynamics by which two oscillating populations in SNr both dynamically predict M1 activity.

\section{M1 is not required for delta oscillations in $\mathrm{SNr}$}

The results of our regression analysis suggest that oscillations in SNr are not caused by $\mathrm{M} 1$, but rather that oscillations in the SNr precede and predict those in M1. To test this hypothesis, we performed M1 aspiration lesions in dopamine depleted mice (Figure 8a) and recorded from the SNr. SNr units in the DD + M1-lesioned mice had similar oscillations to those 
DD mice without M1 lesions (Figure 8b). These mice had a significantly higher fraction of

287

288

oscillating units than control animals, but there was no difference between dopamine depleted animals with or without an M1 lesion (Figure 8c). These results provide additional evidence that M1 is a recipient, not the source, of delta oscillations in DD.

\section{Discussion}

In this paper, we have demonstrated that delta $(0.5-4 \mathrm{~Hz})$, not beta $(7-35 \mathrm{~Hz})$, oscillations are the predominant oscillatory feature in basal ganglia neurons in awake, dopamine depleted mice, and that the fraction of units exhibiting these oscillations is a good marker of dopamine loss and motor deficits. These results are consistent with data from the human PD literature demonstrating that delta oscillations are the dominant or only oscillatory feature in some PD patients (Du et al., 2018; Levy et al., 2002). We further show that these oscillations arise from a loss of action on D2 receptors and that, contrary to conclusions drawn from anesthetized experiments, motor cortex is not required for their generation but rather follows the oscillations evident in the basal ganglia.

\section{A novel method to distinguish oscillations from noise}

Although several studies demonstrate the presence of delta oscillations in the LFP (Levy et al., 2002; Priori et al., 2004) and single units (Steigerwald et al., 2008; Du et al., 2018; Zhuang et al., 2019) of PD patients, many more studies ignore oscillations in this band completely. Difficulties in detecting these oscillations may contribute to this lack of attention. Most studies examining oscillations in PD patients investigate the LFP, not individual spiking units, and the intrinsic low frequency noise of LFP signals makes reliably detecting oscillations in the delta range difficult. Even when it is possible to record from single units, we have demonstrated that low frequency noise can disrupt these spiking signals as well.

To reliably detect low frequency spike oscillations in awake animals, we have introduced phase shift as a novel detection technique which utilizes phase information typically discarded 
312 from the Fourier transform. Phase shift measures the local stationarity of a signal composed

313 primarily of one frequency - a perfect sine wave would have zero phase shift and high power,

314 but a sine wave with a phase that randomly advances would have high phase shift while

315 maintaining high power. This measure can distinguish our signal of interest - a single oscillatory

316 signal that shifts in phase only gradually or rarely - from low frequency pink noise, a

317 phenomenon that is not restricted to a single frequency, for which phase components measured

318 at individual frequencies may shift rapidly between adjacent windows.

319 Relationship to previous studies on PD oscillations

In PD research, much of the oscillation literature has focused on the beta band

321 (Hammond et al., 2007; Jenkinson \& Brown, 2011). Here, we demonstrate dopamine loss and

322 PD-like symptoms in mice without the presence of beta oscillations, and we have previously

323 demonstrated their absence in LFP signals in awake mice as well (Willard et al., 2019). Indeed,

324 to our knowledge, no study has demonstrated the presence of beta oscillations in mouse

325 models of PD. Instead, this study suggests that delta oscillations are an important signal in the

326 dopamine depleted basal ganglia and may cause parkinsonian dysfunction instead of or (in

327 patients or other animal models) alongside beta oscillations.

The low frequency oscillations that we observe resemble those seen in anesthetized mice and rats, although oscillations in awake settings are generally noisier. By performing these experiments in awake mice, this study rules out concerns that oscillations in the basal ganglia

331 are simply entrained by anesthesia-induced oscillations from cortex (Tseng, Kasanetz,

332 Kargieman, Riquelme, et al., 2001; Belluscio et al., 2003) or by artificial respiration devices

333 (Ruskin et al., 2002). Instead, we see that oscillations in the basal ganglia arise even during

334 wakefulness and in fact lead and predict oscillations in M1. While we can rule out one causal

335 direction (M1 -> SNr), it is difficult to know whether SNr entrains M1 directly or if both SNr and

336 M1 are entrained by a common source. 
By referencing SNr oscillations to M1, we distinguish two populations of oscillating SNr neurons. These populations and how they are defined mimic the Type-A (TA) and Type-I (TI) populations observed in GPe whose discharge is high and low, respectively, during the active phase of M1 oscillations (Mallet et al., 2008). Active-predicting (AP) and inactive-predicting (IP)

SNr neurons are a very close analog to TA and TI GPe neurons, respectively, except for two

differences. First, the granularity of our regression analysis illustrates that AP and IP neurons

are not simply active or inactive during the active phase of the M1 oscillation, but begin discharging (AP) or pausing (IP) 150-250 ms before the active component of the M1 oscillation.

345 To our knowledge, a precise timing analysis of TA and TI neurons with M1 oscillations has not been performed to determine if a similar phenomenon occurs in GPe. Second, SNr AP and IP neurons are approximately equal in number, whereas TI neurons are the prevailing population in GPe (72\% TI, 17\% TA) (Mallet et al., 2008). These populations of GPe neurons were later shown to have anatomical (Corbit et al., 2016; Mallet et al., 2012), genetic (Abdi et al., 2015), these analyses would be beyond the scope of our study.

\section{Mechanisms of generation: insight from D2 receptors}

A previous study demonstrated that delta oscillations in anesthetized mice arise we have replicated here in awake mice. This fast onset (<2 minutes) contrasts with the typical

357 longer timescale associated with beta oscillations in DD (Mallet et al., 2008). We further show

358 that these oscillations arise due to a loss of D2R activation and can be ablated in already

359 dopamine depleted animals through D2R agonism. It is unclear where the D2 receptors

360 responsible for this ablation are located, but the high density of D2R's in the striatum make it a

361 strong candidate. Lack of D2R activation causes a wide array of biomolecular changes within

362 D2R-expressing neurons, including the opening of NMDA (Higley \& Sabatini, 2010; Wang et al., 
2012) and L-type calcium channels (Hernández-López et al., 2000), which have been shown to be involved in membrane potential and calcium oscillations, respectively, in other circuits (Guertin \& Hounsgaard, 1998).

In addition to striatum, another candidate for the generation of delta oscillations in DD is the STN-GPe loop. While often associated with beta oscillations (Mallet et al., 2008; NevadoHolgado et al., 2014; Pavlides et al., 2012; Wei et al., 2015), this loop was originally implicated in generating much lower frequency oscillations $(0.8-1.8 \mathrm{~Hz})$ in cultured neurons (Plenz \& Kital, 1999), a phenomenon that has been demonstrated subsequently in computational models

371 (Terman et al., 2002; Modolo et al., 2008). The slow rates associated with the dynamics of T-

372 type calcium channels and of some after-hyperpolarization currents have been shown to contribute the generation of oscillations and could explain the low frequency of these oscillations as well (Devergnas et al., 2015).

\section{Relationship between oscillations and motor dysfunction}

Of those studies that examine low frequency oscillations in PD patients, many consider

377 only their relationship to tremor, seeing both positive and zero correlation with EMG signals

378 during tremor bouts (Hurtado et al., 1999; Du et al., 2018). No study, to our knowledge, has

379 investigated low frequency oscillations in relationship to other PD symptoms. Here, we have established a strong relationship between delta oscillations, dopamine loss, and akinetic dysfunction in mice. Further research and re-examination of existing patient data could elucidate a role for delta oscillations in predicting or causing PD motor deficits in humans.

While we cannot demonstrate a causal link between oscillations and motor dysfunction

384 in this study, it is notable that the emergence of delta oscillations in the SNr from multiple

385 experimental manipulations is consistently paired with a time-locked and commensurate

386 reduction in motor activity. These results suggest a reappraisal of delta oscillations as a

387 potential cause or marker of motor dysfunction in Parkinson's disease patients that could be an 388 underappreciated target for PD therapies. 


\section{Methods}

\section{Animals}

All experiments were conducted in accordance with guidelines from the National Institutes of Health and with approval from the Carnegie Mellon University Institutional Animal Care and Use Committee. Male and female mice on a C57BL/6J background aged 8-15 weeks were randomly allocated into experimental groups (e.g. Control, Bilateral 6OHDA, Reserpine, etc.), except insofar as to ensure that male and female mice were both represented in every group.

\section{Stereotaxic surgery}

Headbar implantation

Animals were anesthetized with $20 \mathrm{mg} / \mathrm{kg}$ ketamine and $6 \mathrm{mg} / \mathrm{kg}$ xylazine and placed in a stereotaxic frame (Kopf Instruments). Anesthesia was maintained throughout surgery with 1.0and DV relative to the dural surface. The scalp was opened and bilateral craniotomies (for later probe insertion) approximately $1.5 \times 1.5 \mathrm{~mm}$ in size were drilled over SNr (AP: -3.00, ML:

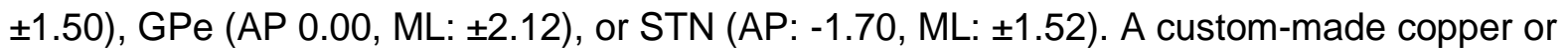
stainless steel headbar was affixed to the mouse's skull with dental cement (Lang Dental). A well of dental cement was then built around the exposed skull (see in vivo recordings) and filled

408 with a silicon elastomer.

Dopamine depletion

A hole was drilled on one (for unilateral) or both (for bilateral) hemispheres of the skull

411 over the medial forebrain bundle (MFB, AP: $-0.80, M L: \pm 1.10)$. A unilateral infusion cannula

412 (PlasticsOne) was slowly lowered into the brain $5 \mathrm{~mm}$ below the dura. $1 \mu \mathrm{L}$ of $5 \mu \mathrm{g} / \mu \mathrm{L} 6 \mathrm{OHDA}$

413 (Sigma-Aldrich) or $0.9 \%$ saline was injected over the course of 5 minutes with a GenieTouch 414 Hamilton syringe pump (Kent Scientific). The infusion cannula was left in place for 5 minutes 
415 post-injection before being slowly retracted. For animals undergoing bilateral depletion, this

416 process was repeated on the opposite hemisphere.

417 Cannula implantation

For experiments involving acute drug infusion into the MFB or gradual dopamine depletion with 6OHDA, a bilateral guide cannula (Plastics One) was implanted (same coordinates as dopamine depletion) using dental cement (Lang Dental) and a dummy cannula was placed in the guide. Before infusion, the dummy was replaced with an infusion cannula and

422 attached to the same Hamilton syringe pump as above. Gradually depleted animals were 423 infused with $1 \mu \mathrm{L}$ of $0.75 \mu \mathrm{g} / \mu \mathrm{L}$ 6OHDA every 5 days (See Willard et al 2019 for full details). ECoG connector implantation (Ampityco Electronics) was soldered to a stainless steel wire, and the connector was gently

427 lowered above left or right motor cortex (M1, AP: $+1.40, \mathrm{ML}: \pm 1.00)$ such that the wire touched

428 the dural surface then secured in place with dental cement (Lang Dental). attached to a vacuum source, we aspirated cortex to a depth of $2.5 \mathrm{~mm}$ across the craniotomy 433 and under portions of the remaining skull, periodically lightly rinsing the area with saline. We

434 filled the lesioned space with triple antibiotic (bacitracin, neomycin, polymyxin) before sealing 435 the craniotomy with a silicon elastomer (Smooth-On)

436 Post-operative care

Upon completion of surgery, animals were injected subcutaneously with $0.5 \mathrm{mg} / \mathrm{kg}$

438 ketofen and placed inside their cage half on/half off a heating pad to recover. Dopamine 439 depleted animals were supplied with trail mix and moistened food to maintain weight and 
440 hydration, in addition to their usual food pellets and water bottles, and animals were tracked

441 regularly to ensure proper health and weight.

\section{Drugs}

In addition to the drugs used above during surgery, animals were given the following drugs (Sigma-Aldrich, except when specified) dissolved in $0.9 \%$ saline (except when specified).

446 For reserpine depletions, animals were injected i.p. daily for three days with $5 \mathrm{mg} / \mathrm{kg}$ reserpine

447 in $2 \%$ acetic acid (diluted in $0.9 \%$ saline). For recordings involving dopamine agonists and antagonists, animals were injected i.p. during recording with either $0.4 \mathrm{mg} / \mathrm{kg} \mathrm{SCH} 22390$, 3 mg/kg raclopride. $1 \mathrm{mg} / \mathrm{kg}$ SKF81297 (Tocris Biosciences), or $3 \mathrm{mg} / \mathrm{kg}$ quinpirole. Acute infusions into the MFB used $2 \%$ lidocaine.

\section{In vivo recordings}

Mice were head-fixed atop a free-running wheel (Heiney et al., 2014). After acclimation

454 to head-fixation for ten minutes, the silicon elastomer was removed and craniotomies were cleaned with saline. Using a micromanipulator (Sutter Instruments), a linear microelectrode probe with sixteen channels spaced $50 \mu \mathrm{m}$ apart (NeuroNexus) was lowered into the craniotomy

457 at the coordinates listed above for SNr, GPe or STN. After the initial lowering, a ground wire was placed in saline in the dental cement well on the skull. Once the top of the nucleus (SNr, 4.0mm, GPe: $-3.60 \mathrm{~mm}$, STN: $-4.00 \mathrm{~mm}$ from the top of the brain) was found and high firing rate

460 units were observed, the probe was held stable for at least ten minutes prior to recording.

461 Spiking (bandpass filtered for $150-8000 \mathrm{~Hz}$, sampled at $40 \mathrm{kHz}$ ) and local field potential

462 (bandpass filtered to $0.5-300 \mathrm{~Hz}$, sampled at $1 \mathrm{kHz}$ ) recordings were collected through an

463 OmniPlex amplifier (Plexon, Inc.) with common median virtual referencing. After recording for at

464 least three minutes, the probe was lowered to explore the full dorsal-ventral extent of the 465 nucleus. Simultaneous to these recordings, the mouse's walking speed on the wheel was 
recorded using an optical mouse and fed to a TTL-pulser which was connected to the OmniPlex amplifier analog input. For ECoG recordings, the gold implant was connected to a headstage with a ground wire in saline on top of the skull. The headstage was connected to an amplifier (AM Systems) with 1000x gain and $0.1-500 \mathrm{~Hz}$ bandpass filtering and this amplifier was connected to the OmniPlex amplifier analog input.

\section{Histology}

After recording, animals were sacrificed and perfused with $4 \%$ paraformaldehyde (PFA).

481 (for unilateral 6OHDA injection) to be considered for analysis. and an Iba1 antibody (rabbit antimagnification (Keyence BZ-X) and outlines of nuclei of interest were overlaid on the images

485 (from Paxinos Mouse Brain Atlas in Stereotaxic Coordinates, Second Edition).

\section{Data pre-processing}

Spikes were manually sorted into single units using Offline Sorter (Plexon). For

490 waveforms generated a cluster of spikes significantly distinct from other unit or noise clusters ( $p$ $491<.05), 2)$ the J3-statistic was greater than 1, 3) the Davies-Bouldin statistic was less than 0.5 , 
492

493

494

495

496

497

498

499

500

501

502

503

504

505

506

507

508

509

510

511

512

513

514

515

and 4 ) fewer than $0.15 \%$ of ISl's were less than $2 \mathrm{~ms}$. In the case where a unit was lost during recording, it was only used in analysis for the time period when its spike cluster satisfied these criteria, and only if its cluster was present for at least three minutes. Data were then imported into MATLAB (MathWorks) in which all further analysis was performed using custom code except when specified.

Since units must fire quickly enough to exhibit an oscillation, only units with a firing rate greater than $5 \mathrm{~Hz}$ (over 95\% of sorted units) were considered for analysis. As ECoG signals were occasionally corrupted for short time windows, generally due to muscle activity, we visually determined a noise threshold for each recording and zeroed any length of signal within 250 milliseconds of any data point whose absolute value exceeded that threshold. ECoG signals were then delta $(0.5-4 \mathrm{~Hz})$ bandpassed using a $2^{\text {nd }}$ order Butterworth filter.

\section{Oscillation detection and visualization}

Renewal-Corrected Power Spectrum

For each unit, we downsampled its spike train to $1 \mathrm{kHz}$ and split it into segments of $2^{12}$

$\mathrm{ms}$, advancing from one segment to the next with time step size $\Delta s=2^{9} \mathrm{~ms}$. For each segment, we calculated its interspike interval (ISI) probability distribution, $P_{0}(t)$. We calculated $\hat{C}_{0}(\omega)$, the theoretical power spectral density (PSD) of a renewal process defined by $P_{0}(t)$ scaled by the number of spikes in the segment:

$$
\hat{C}_{0}(\omega)=\operatorname{Re}\left(\frac{1+\hat{P}_{0}(\omega)}{1-\hat{P}_{0}(\omega)}\right) n
$$

where $\operatorname{Re}(x)$ indicates the real part of $\mathrm{x}, \hat{P}_{0}(\omega)$ indicates the Fourier transform of the ISI distribution in appropriate frequency units, and $\mathrm{n}$ is the number of spikes in the segment. This is a variant of a method presented previously for calculating $\hat{C}_{0}(\omega)$ analytically rather than approximating it through Monte Carlo shuffling simulations (Rivlin-Etzion et al., 2006). 
We next calculated an estimate of the PSD of the spike train in that segment: ${ }^{1}$

$$
\hat{C}_{\infty}(\omega)=|F F T(x(t))|^{2}
$$

518 where $x(t)$ is the mean-subtracted spike train in the segment, FFT is the fast Fourier transform

519 (MATLAB function $\mathrm{fft}$ ) and vertical bars indicate absolute value. Finally, we normalized this estimate to achieve the renewal-corrected PSD of a single segment:

$$
\hat{C}(\omega)=\frac{\hat{C}_{\infty}(\omega)}{\hat{C}_{0}(\omega)}
$$
brevity.

Phase Shift

For the kth time segment, we calculated the uncorrected phase $\tilde{\phi}$ at each frequency:

$$
\tilde{\phi}(\omega, k)=\tan ^{-1}(F F T(x(t)))
$$

528 and made the following correction such that the phase of each frequency is defined relative to

529 the start of the recording rather than the start of the segment:

$$
\phi(\omega, k)=\bmod (\pi+(\tilde{\phi}-2 \pi \omega(k-1) \Delta s), 2 \pi)-\pi
$$

531 where mod is the modulus operator and $\Delta s$ is the time step between adjacent segments (here,

$5322 \% 1000$ seconds). In other words, for each frequency, imagine a perfect oscillator with zero

533 phase at the start of the recording. For each segment, we determined what phase this oscillator

534 would reach at the start of the segment and defined that phase to be zero for that segment. This

535 correction ensures that a perfect oscillator would have the same corrected phase $\phi$ for every

536 segment.

\footnotetext{
${ }^{1}$ Note that a rectangular window is used throughout this section. This is because 1) compared to tapered windows, the rectangular window's maximal frequency resolution ensures a peak in the PSD representing an oscillation of interest will not mix with nearby peaks caused by pink noise, and 2) multiplication with a window function manifests as a convolution in the frequency domain, which distorts phase and yields a nearly flat and uninformative phase shift plot.
} 
After computing the corrected phase of all segments, we approximated the time

538

\section{Oscillation Detection}

We detected oscillations in a two-step process by first seeking frequencies with high power and then determining whether these frequencies also had low phase shift.

To determine whether a unit reached statistically significantly high power at a particular frequency, we found each local maximum of $\hat{C}(\omega)$, defined as a value higher than its three neighbors on both sides, within the band $0.5-4 \mathrm{~Hz}$ (or 7-35 Hz for detecting beta oscillations). We then estimated a 99\% confidence interval of renewal-corrected power from the region of $\hat{C}(\omega)$ between 100 and $500 \mathrm{~Hz}$, correcting for multiple comparisons (Bonferroni correction) of all frequencies in the band of interest. A peak of $\hat{C}(\omega)$ was considered significant if it fell above this confidence interval.

As our second step, we determined if any frequency detected in the previous step had a significantly low phase shift. We estimated a 95\% confidence interval of phase shifts from the region of $\xi(\omega)$ between 100 and $500 \mathrm{~Hz}$, correcting for multiple comparisons (Bonferroni correction) if multiple frequencies were detected from the PSD. We concluded that an oscillation was present at a frequency with significant power if the phase shift at that frequency fell below this confidence interval.

\section{Spike Spectrograms}


For time frequency analyses, the process outlined under Renewal-Corrected Power

562 Spectrum was modified to use segments of length $2^{13} \mathrm{~ms}$ with $2^{11} \mathrm{~ms}$ overlap to improve

563 visualization. Rather than averaging over segments, the resultant matrix was smoothed with a

564 3×3 2-D gaussian filter and plotted as a normalized heatmap (MATLAB function imagesc). Due

565 to the loss of fine frequency resolution at low frequencies, this procedure was only used on

566 spike trains in which an oscillation was detected in the previous procedure.

\section{Neural Measures}

Beyond oscillations, we investigated several other neural measures - firing rate, firing variability, bursts and synchrony. A unit's firing rate was defined as its number of spikes divided by the total time of recording. Variability was measured as the coefficient of variation (standard

572 deviation divided by mean) of a unit's interspike intervals. Bursts were quantified using the

573 Poisson surprise algorithm (Legendy \& Salcman, 1985) with a surprise threshold of 5, initial

574 firing rate threshold of $200 \%$ of baseline calculated over the entire recording, and removal of

575 any burst with fewer than 3 spikes.

To determine if two units were synchronous, we used the method and parameters

577 outlined in Willard et al. 2019, which determines the fraction of synchronous spikes above 578 chance after correcting for nonstationarity in a unit's firing rate (Willard et al., 2019). In brief, we 579 windowed both spike trains into 12-second segments and zeroed the first and last four seconds 580 of the segment taken from the second spike train. We performed cross-correlation with a 581 maximum lag of four seconds. Since this maximum lag is equal to the length of time zeroed on 582 the second spike train, this ensures a constant number of non-zero-padded comparisons $\left(n_{c}\right)$ at 583 each lag, as opposed to traditional cross-correlation in which $n_{c}$ is a function of lag. We divided 584 the cross-correlogram for the segment by the mean value from $0.5-4$ seconds on both sides, 585 which allows the correlation's units to be interpreted as the fraction of spikes greater than 586 chance at a given lag (where $1=$ chance). We repeated this process on overlapping segments 
587 (time step $=4$ seconds) and then averaged these results together to get the mean,

588 nonstationarity-corrected cross-correlogram. We generated a 99\% confidence interval from the

589 data with lag $\geq 0.5 \mathrm{~s}$ (which is a reasonable null distribution due to $n_{c}$, and thus the variance of

590 the correlation estimate, being held constant). We conclude that a pair is synchronous if its

591 normalized cross-correlation at lag zero is larger than the upper boundary of this confidence

592 interval.

593

\section{Behavioral Testing and Metric}

Full details on the behavioral testing and the principal component analysis (PCA) metric

596 for gradually depleted animals can be found in Willard et al. 2019. In brief, PCA was performed

597 on the following metrics from behavioral tests: mean speed in an open field, number of rears in

59810 minutes in a small enclosure, total time spent traversing a pole task, and latency to fall on a

599 wire hang task.

600

601

\section{Linear Regression}

602

Linear regression was performed using ordinary least squares. To determine if a linear fit was statistically significant, we computed 1000 fits each using a random subsample containing $80 \%$ of the data. We computed a bootstrapped confidence interval of the slope of this linear relationship from the middle $99 \%$ of the slopes of these 1000 fits, and the relationship was considered significant if this interval did not include zero.

\section{Decision Tree Regression}

We sought to determine the relationship between dopamine loss, motor symptoms and

610 neural firing by predicting animals' TH immunofluorescence (see Histology) and the first

611 principal component (PC1) of their behavior (see Behavioral Testing and Metric) from four

612 physiological measures (see Neural Measures) and prevalence of delta oscillations. Firing rate, 
613 CV and bursts/second were averaged across all neurons for each animal, synchrony was

614 measured as the fraction of synchronous pairs of units, and oscillations were measured as the

615 fraction of delta oscillating units. Because of the highly nonlinear nature of these parameters'

616 relationships to dopamine loss and behavior (Willard et al., 2019), we used a variant of decision

617 tree regression, a highly nonlinear regression method.

618 We built an individual tree on $80 \%$ of the data (20 animals) using the fit method of the

619 DecisionTreeRegressor class in the scikit-learn package for Python to predict the percent of TH

620 remaining $(\mathrm{Y})$ from the above neuronal parameters (a set $\mathrm{X}$ ). In brief, this method places all

621 training data at the topmost node of a tree and calculates the mean squared error (MSE) of this

622 node as if each animal's TH were estimated to be the mean TH of every animal at the node. We

623 determined, for each parameter $\mathrm{X}$, the threshold $\mathrm{T}$ that would most reduce the mean squared

624 error (MSE) of the animals if they were to be estimated in two different sets depending on

625 whether their value of X is "greater than" or "less than or equal to" T. We then found the

626 parameter for which the best T most reduces that MSE and split the animals at that node into

627 two new child nodes according to the identified threshold. We iteratively repeated this process

628 at every node until all terminal nodes had two or fewer animals at them, at which point each

629 terminal node is termed a "leaf" of the tree.

We tested the remaining $20 \%$ of the data (5 animals) using the DecisionTreeRegressor

631 test method, which runs each animal through the tree (picking $>$ or $\leq$ at each node as

632 determined by the animal's data) until it reaches a leaf. The mean value of $Y$ at each leaf is the

633 prediction for that animal. We computed the error of the tree as the root-mean-squared error

634 (RMSE) of its 5 predictions.

We computed a forest of 1000 such trees through subsampling the data into training and

636 testing sets (Monte Carlo cross-validation) and calculated the top and bottom 2.5 percentiles to

637 approximate a 95\% confidence interval for the forest. We generated an intercept-only forest

638 (using no parameters in the training set) and oscillation-only forest (using only the fraction of 
oscillations and an intercept term in the training set) on the same 1000 bootstrapped training and testing sets.

The importance of each parameter was determined using a variant on permutation importance. For a given parameter and tree, consider the set $\mathrm{S}$ of values for that parameter in the test set. We produced pseudo-test data with every derangement of S (i.e. 5 animals $\times 44$ derangements of 5 values $=220$ pseudo-test animals with shuffled data for one parameter). The difference between the RMSE of the real test data and the pseudo-test data is the importance of that parameter for that tree. To determine the parameter importance for the entire forest, we approximate a $95 \%$ confidence intervals as above from the 1000 trees.

A forest predicting the first principal component (PC1) of behavior instead of \% $\mathrm{TH}$ remaining was computed in the same manner.

\section{ECoG-Spike Time Series Regression}

To determine if SNr neurons have a significant lead/lag relationship with $\mathrm{M} 1$, we built a series of regression models predicting an M1 ECoG signal from the spiking of a single SNr unit at various lags. First, we binned the ECoG into $10 \mathrm{~ms}$ bins and defined the dependent variable $Y$ as the difference between adjacent ECoG measurements to reduce nonstationarity. We then built a 10th order autoregressive model of $Y$ which served as the null model.

To incorporate SNr firing into the prediction, we calculated the spike density function (SDF) for an SNr unit by convolving its spike train with a Gaussian function with a standard deviation of $100 \mathrm{~ms}$. We then aimed to determine which time shift of the SDF best improves the prediction of the ECoG. One might use a distributed lag model for this task, where the explanatory variables consist of the time shifted ECoG (autoregression) and all considered time shifts of the SNr SDF simultaneously in a single model, but the multicollinearity of the SDF at different time shifts can heavily bias the regression coefficients. Instead we assumed that, if a lag exists by which the unit firing influences the ECoG or vice versa, then there is only one such 
665

666

667

668

669

670

671

672

673

674

675

676

677

678

679

680

681

682

683

684

685

686

687

688

689

690

lag by which this influence occurs. Thus, we could build an individual model for each time shift of the SDF. Each model used the $10^{\text {th }}$ order autoregressive terms and one SDF term shifted from between -100 and +100 bins $(-1000$ to $+1000 \mathrm{~ms})$ as its explanatory variables. We built 201 such models, which covers the entire range of lags at 1 bin increments.

To determine if a significant lead/lag existed, we found the best model as determined by its mean squared error (MSE). We then determined if the model at this lag was significantly better than the null autoregressive model by performing an F-test at $\alpha<0.05$, correcting for 201 comparisons (Bonferroni correction). As choosing ECoG as the independent variable and using autoregressive terms from the past could introduce bias in favor of SNr predicting M1, we also performed these analyses using SNr as the independent variable (i.e. computing a null autoregressive model for SNr spiking and then computing 201 models at distinct ECoG time shifts to compare to the null), and performed the same analysis as above but in backwards time (i.e. building an autoregressive model of the ECoG from future ECoG samples). These analyses gave very similar results to the original analysis but were omitted for brevity.

\section{Statistical Tests}

Statistical tests were performed to establish if fractions of oscillatory units and fractional ECoG bandpowers were significantly different across conditions. For comparisons with two groups, a two-sample t-test was performed, unless data were paired before and after a manipulation (e.g. acute drug infusion), in which case a one-sample t-test was performed. For comparisons with multiple groups compared against a control group, a one-way ANOVA was performed, and if this reached significance at the $\alpha=0.05$ level, a Dunnett's post-hoc test was performed to determine if there were individual differences comparing groups to control. Asterisks above comparisons in figures correspond to ${ }^{*}: p<0.05,{ }^{* *}: p<0.01,{ }^{* *} p<0.0001$. 


\section{Acknowledgements}

692 Thanks to Rachel Bouchard, Hyun Young Park, Jenna Schwenk, Christen Snyder and Robert S.

693 Turner for their help with this project. This work was supported by NSF awards DMS 1516288

694 (AHG, JER), 1724240 (JER), and NIH awards R01NS101016, R01NS104835, and

695 R21NS095103 (AHG), and F31NS101821 (TCW)

696

\section{Competing Interests}

698 No competing interests to declare. 


\section{References}

Abdi, A., Mallet, N., Mohamed, F. Y., Sharott, A., Dodson, P. D., Nakamura, K. C., Suri, S., Avery, S. V., Larvin, J. T., Garas, F. N., Garas, S. N., Vinciati, F., Morin, S., Bezard, E., Baufreton, J., \& Magill, P. J. (2015). Prototypic and Arkypallidal Neurons in the Dopamine-Intact External Globus Pallidus. Journal of Neuroscience, 35(17), 6667-6688. https://doi.org/10.1523/JNEUROSCI.4662-14.2015

Aristieta, A., Ruiz-Ortega, J. A., Miguelez, C., Morera-Herreras, T., \& Ugedo, L. (2016). Chronic L-DOPA administration increases the firing rate but does not reverse enhanced slow frequency oscillatory activity and synchronization in substantia nigra pars reticulata neurons from 6hydroxydopamine-lesioned rats. Neurobiology of Disease, 89(Supplement C), 88-100. https://doi.org/10.1016/j.nbd.2016.02.003

Belluscio, M. A., Kasanetz, F., Riquelme, L. A., \& Murer, M. G. (2003). Spreading of slow cortical rhythms to the basal ganglia output nuclei in rats with nigrostriatal lesions. European Journal of Neuroscience, 17(5), 1046-1052. https://doi.org/10.1046/j.1460-9568.2003.02543.x

Bergman, H., Wichmann, T., Karmon, B., \& DeLong, M. R. (1994). Parkinsonian Tremor is Associated with Low Frequency Neuronal Oscillations in Selective Loops of the Basal Ganglia. In G. Percheron, J. S. McKenzie, \& J. Féger (Eds.), The Basal Ganglia IV: New Ideas and Data on Structure and Function (pp. 317-325). Springer US. https://doi.org/10.1007/978-1-4613-0485-2_33

Connolly, A. T., Jensen, A. L., Bello, E. M., Netoff, T. I., Baker, K. B., Johnson, M. D., \& Vitek, J. L. (2015). Modulations in Oscillatory Frequency and Coupling in Globus Pallidus with Increasing Parkinsonian Severity. Journal of Neuroscience, 35(15), 6231-6240. https://doi.org/10.1523/JNEUROSCI.4137-14.2015

Corbit, V. L., Whalen, T. C., Zitelli, K. T., Crilly, S. Y., Rubin, J. E., \& Gittis, A. H. (2016). Pallidostriatal Projections Promote $\beta$ Oscillations in a Dopamine-Depleted Biophysical Network Model. Journal of Neuroscience, 36(20), 5556-5571. https://doi.org/10.1523/JNEUROSCI.0339-16.2016 
Devergnas, A., Chen, E., Ma, Y., Hamada, I., Pittard, D., Kammermeier, S., Mullin, A. P., Faundez, V., Lindsley, C. W., Jones, C., Smith, Y., \& Wichmann, T. (2015). Anatomical localization of Cav3.1 calcium channels and electrophysiological effects of T-type calcium channel blockade in the motor thalamus of MPTP-treated monkeys. Journal of Neurophysiology, 115(1), 470-485. https://doi.org/10.1152/jn.00858.2015

Du, G., Zhuang, P., Hallett, M., Zhang, Y.-Q., Li, J.-Y., \& Li, Y.-J. (2018). Properties of oscillatory neuronal activity in the basal ganglia and thalamus in patients with Parkinson's disease. Trans/ational Neurodegeneration, 7(1), 17. https://doi.org/10.1186/s40035-018-0123-y

Foffani, G., Ardolino, G., Egidi, M., Caputo, E., Bossi, B., \& Priori, A. (2006). Subthalamic oscillatory activities at beta or higher frequency do not change after high-frequency DBS in Parkinson's disease. Brain Research Bulletin, 69(2), 123-130. https://doi.org/10.1016/j.brainresbull.2005.11.012

Gage, G. J., Stoetzner, C. R., Wiltschko, A. B., \& Berke, J. D. (2010). Selective Activation of Striatal FastSpiking Interneurons during Choice Execution. Neuron, 67(3), 466-479. https://doi.org/10.1016/j.neuron.2010.06.034

Galati, S., D’Angelo, V., Olivola, E., Marzetti, F., Di Giovanni, G., Stanzione, P., \& Stefani, A. (2010). Acute inactivation of the medial forebrain bundle imposes oscillations in the SNr: A challenge for the 6OHDA model? Experimental Neurology, 225(2), 294-301. https://doi.org/10.1016/j.expneurol.2010.06.020

Guertin, P. A., \& Hounsgaard, J. (1998). NMDA-Induced Intrinsic Voltage Oscillations Depend on L-Type Calcium Channels in Spinal Motoneurons of Adult Turtles. Journal of Neurophysiology, 80(6), 3380-3382. https://doi.org/10.1152/jn.1998.80.6.3380 
Halje, P., Brys, I., Mariman, J. J., da Cunha, C., Fuentes, R., \& Petersson, P. (2019). Oscillations in corticobasal ganglia circuits: Implications for Parkinson's disease and other neurologic and psychiatric conditions. Journal of Neurophysiology, 122(1), 203-231. https://doi.org/10.1152/jn.00590.2018

Hammond, C., Bergman, H., \& Brown, P. (2007). Pathological synchronization in Parkinson's disease: Networks, models and treatments. Trends in Neurosciences, 30(7), 357-364. https://doi.org/10.1016/j.tins.2007.05.004

Heimer, G., Rivlin, M., Israel, Z., \& Bergman, H. (2006). Synchronizing activity of basal ganglia and pathophysiology of Parkinson's disease. In P. Riederer, H. Reichmann, M. B. H. Youdim, \& M. Gerlach (Eds.), Parkinson's Disease and Related Disorders (pp. 17-20). Springer. https://doi.org/10.1007/978-3-211-45295-0_4

Heiney, S. A., Wohl, M. P., Chettih, S. N., Ruffolo, L. I., \& Medina, J. F. (2014). Cerebellar-Dependent Expression of Motor Learning during Eyeblink Conditioning in Head-Fixed Mice. Journal of Neuroscience, 34(45), 14845-14853. https://doi.org/10.1523/JNEUROSCI.2820-14.2014

Hernández-López, S., Tkatch, T., Perez-Garci, E., Galarraga, E., Bargas, J., Hamm, H., \& Surmeier, D. J. (2000). D2 Dopamine Receptors in Striatal Medium Spiny Neurons Reduce L-Type Ca2+ Currents and Excitability via a Novel PLCß1-IP3-Calcineurin-Signaling Cascade. Journal of Neuroscience, 20(24), 8987-8995. https://doi.org/10.1523/JNEUROSCI.20-24-08987.2000

Higley, M. J., \& Sabatini, B. L. (2010). Competitive regulation of synaptic Ca $2+$ influx by D2 dopamine and A2A adenosine receptors. Nature Neuroscience, 13(8), 958-966. https://doi.org/10.1038/nn.2592

Hurtado, J. M., Gray, C. M., Tamas, L. B., \& Sigvardt, K. A. (1999). Dynamics of tremor-related oscillations in the human globus pallidus: A single case study. Proceedings of the National Academy of Sciences, 96(4), 1674-1679. https://doi.org/10.1073/pnas.96.4.1674 
Jenkinson, N., \& Brown, P. (2011). New insights into the relationship between dopamine, beta oscillations and motor function. Trends in Neurosciences, 34(12), 611-618. https://doi.org/10.1016/j.tins.2011.09.003

Kühn, A. A., Kempf, F., Brücke, C., Doyle, L. G., Martinez-Torres, I., Pogosyan, A., Trottenberg, T., Kupsch, A., Schneider, G.-H., Hariz, M. I., Vandenberghe, W., Nuttin, B., \& Brown, P. (2008). HighFrequency Stimulation of the Subthalamic Nucleus Suppresses Oscillatory $\beta$ Activity in Patients with Parkinson's Disease in Parallel with Improvement in Motor Performance. Journal of Neuroscience, 28(24), 6165-6173. https://doi.org/10.1523/JNEUROSCI.0282-08.2008

Kühn, A. A., Kupsch, A., Schneider, G.-H., \& Brown, P. (2006). Reduction in subthalamic 8-35 Hz oscillatory activity correlates with clinical improvement in Parkinson's disease. European Journal of Neuroscience, 23(7), 1956-1960. https://doi.org/10.1111/j.1460-9568.2006.04717.x

Legendy, C. R., \& Salcman, M. (1985). Bursts and recurrences of bursts in the spike trains of spontaneously active striate cortex neurons. Journal of Neurophysiology, 53(4), 926-939. https://doi.org/10.1152/jn.1985.53.4.926

Lenz, F. A., Tasker, R. R., Kwan, H. C., Schnider, S., Kwong, R., Murayama, Y., Dostrovsky, J. O., \& Murphy, J. T. (1988). Single unit analysis of the human ventral thalamic nuclear group: Correlation of thalamic "tremor cells" with the 3-6 Hz component of parkinsonian tremor. Journal of Neuroscience, 8(3), 754-764. https://doi.org/10.1523/JNEUROSCI.08-03-00754.1988

Levy, R., Ashby, P., Hutchison, W. D., Lang, A. E., Lozano, A. M., \& Dostrovsky, J. O. (2002). Dependence of subthalamic nucleus oscillations on movement and dopamine in Parkinson's disease. Brain, 125(6), 1196-1209. https://doi.org/10.1093/brain/awf128

Magill, P. J., Bolam, J. P., \& Bevan, M. D. (2001). Dopamine regulates the impact of the cerebral cortex on the subthalamic nucleus-globus pallidus network. Neuroscience, 106(2), 313-330. https://doi.org/10.1016/S0306-4522(01)00281-0 
Mallet, N., Micklem, B. R., Henny, P., Brown, M. T., Williams, C., Bolam, J. P., Nakamura, K. C., \& Magill, P. J. (2012). Dichotomous Organization of the External Globus Pallidus. Neuron, 74(6), 10751086. https://doi.org/10.1016/j.neuron.2012.04.027

Mallet, N., Pogosyan, A., Márton, L. F., Bolam, J. P., Brown, P., \& Magill, P. J. (2008). Parkinsonian Beta Oscillations in the External Globus Pallidus and Their Relationship with Subthalamic Nucleus Activity. Journal of Neuroscience, 28(52), 14245-14258. https://doi.org/10.1523/JNEUROSCl.4199-08.2008

Mallet, N., Schmidt, R., Leventhal, D., Chen, F., Amer, N., Boraud, T., \& Berke, J. D. (2016). Arkypallidal Cells Send a Stop Signal to Striatum. Neuron, 89(2), 308-316. https://doi.org/10.1016/j.neuron.2015.12.017

McCairn, K. W., \& Turner, R. S. (2009). Deep Brain Stimulation of the Globus Pallidus Internus in the Parkinsonian Primate: Local Entrainment and Suppression of Low-Frequency Oscillations. Journal of Neurophysiology, 101(4), 1941-1960. https://doi.org/10.1152/jn.91092.2008

McConnell, G. C., So, R. Q., Hilliard, J. D., Lopomo, P., \& Grill, W. M. (2012). Effective Deep Brain Stimulation Suppresses Low-Frequency Network Oscillations in the Basal Ganglia by Regularizing Neural Firing Patterns. Journal of Neuroscience, 32(45), 15657-15668. https://doi.org/10.1523/JNEUROSCI.2824-12.2012

Modolo, J., Henry, J., \& Beuter, A. (2008). Dynamics of the Subthalamo-pallidal Complex in Parkinson's Disease During Deep Brain Stimulation. Journal of Biological Physics, 34(3-4), 251-266. https://doi.org/10.1007/s10867-008-9095-y

Muralidharan, A., Jensen, A. L., Connolly, A., Hendrix, C. M., Johnson, M. D., Baker, K. B., \& Vitek, J. L. (2016). Physiological changes in the pallidum in a progressive model of Parkinson's disease: Are oscillations enough? Experimental Neurology, 279(Supplement C), 187-196.

https://doi.org/10.1016/j.expneurol.2016.03.002 
Nevado-Holgado, A. J., Mallet, N., Magill, P. J., \& Bogacz, R. (2014). Effective connectivity of the subthalamic nucleus-globus pallidus network during Parkinsonian oscillations. The Journal of Physiology, 1429-1455. https://doi.org/10.1113/jphysiol.2013.259721@10.1002/(ISSN)14697793(CAT)Virtuallssues(VI)SfN2014

Parr-Brownlie, L. C., Poloskey, S. L., Bergstrom, D. A., \& Walters, J. R. (2009). Parafascicular thalamic nucleus activity in a rat model of Parkinson's disease. Experimental Neurology, 217(2), 269-281. https://doi.org/10.1016/j.expneurol.2009.02.010

Pavlides, A., Hogan, S. J., \& Bogacz, R. (2012). Improved conditions for the generation of beta oscillations in the subthalamic nucleus-globus pallidus network. European Journal of Neuroscience, 36(2), 2229-2239. https://doi.org/10.1111/j.1460-9568.2012.08105.x

Plenz, D., \& Kital, S. T. (1999). A basal ganglia pacemaker formed by the subthalamic nucleus and external globus pallidus. Nature, 400(6745), 677-682. https://doi.org/10.1038/23281

Priori, A., Foffani, G., Pesenti, A., Tamma, F., Bianchi, A. M., Pellegrini, M., Locatelli, M., Moxon, K. A., \& Villani, R. M. (2004). Rhythm-specific pharmacological modulation of subthalamic activity in Parkinson's disease. Experimental Neurology, 189(2), 369-379. https://doi.org/10.1016/j.expneurol.2004.06.001

Ray, N. J., Jenkinson, N., Wang, S., Holland, P., Brittain, J. S., Joint, C., Stein, J. F., \& Aziz, T. (2008). Local field potential beta activity in the subthalamic nucleus of patients with Parkinson's disease is associated with improvements in bradykinesia after dopamine and deep brain stimulation. Experimental Neurology, 213(1), 108-113. https://doi.org/10.1016/j.expneurol.2008.05.008 Raz, A., Vaadia, E., \& Bergman, H. (2000). Firing Patterns and Correlations of Spontaneous Discharge of Pallidal Neurons in the Normal and the Tremulous 1-Methyl-4-Phenyl-1,2,3,6Tetrahydropyridine Vervet Model of Parkinsonism. Journal of Neuroscience, 20(22), 8559-8571. https://doi.org/10.1523/JNEUROSCI.20-22-08559.2000 
840

841

842

843

844

845

846

847

848

849

850

851

852

853

854

855

856

857

858

859

860

861

862

Rivlin-Etzion, M., Ritov, Y., Heimer, G., Bergman, H., \& Bar-Gad, I. (2006). Local Shuffling of Spike Trains Boosts the Accuracy of Spike Train Spectral Analysis. Journal of Neurophysiology, 95(5), 32453256. https://doi.org/10.1152/jn.00055.2005

Rossi, L., Marceglia, S., Foffani, G., Cogiamanian, F., Tamma, F., Rampini, P., Barbieri, S., Bracchi, F., \& Priori, A. (2008). Subthalamic local field potential oscillations during ongoing deep brain stimulation in Parkinson's disease. Brain Research Bulletin, 76(5), 512-521. https://doi.org/10.1016/j.brainresbull.2008.01.023

Ruskin, D. N., Bergstrom, D. A., \& Walters, J. R. (2002). Nigrostriatal Lesion and Dopamine Agonists Affect Firing Patterns of Rodent Entopeduncular Nucleus Neurons. Journal of Neurophysiology, 88(1), 487-496. https://doi.org/10.1152/jn.00844.2001

Steigerwald, F., Pötter, M., Herzog, J., Pinsker, M., Kopper, F., Mehdorn, H., Deuschl, G., \& Volkmann, J. (2008). Neuronal Activity of the Human Subthalamic Nucleus in the Parkinsonian and Nonparkinsonian State. Journal of Neurophysiology, 100(5), 2515-2524. https://doi.org/10.1152/jn.90574.2008

Swan, C. B., Schulte, D. J., Brocker, D. T., \& Grill, W. M. (2019). Beta frequency oscillations in the subthalamic nucleus are not sufficient for the development of symptoms of parkinsonian bradykinesia/akinesia in rats. ENeuro, ENEURO.0089-19.2019. https://doi.org/10.1523/ENEURO.0089-19.2019

Terman, D., Rubin, J. E., Yew, A. C., \& Wilson, C. J. (2002). Activity Patterns in a Model for the Subthalamopallidal Network of the Basal Ganglia. Journal of Neuroscience, 22(7), 2963-2976.

Tort, A. B. L., Ponsel, S., Jessberger, J., Yanovsky, Y., Brankačk, J., \& Draguhn, A. (2018). Parallel detection of theta and respiration-coupled oscillations throughout the mouse brain. Scientific Reports, 8(1), 1-14. https://doi.org/10.1038/s41598-018-24629-z 
Tseng, K. Y., Kasanetz, F., Kargieman, L., Pazo, J. H., Murer, M. G., \& Riquelme, L. A. (2001). Subthalamic nucleus lesions reduce low frequency oscillatory firing of substantia nigra pars reticulata neurons in a rat model of Parkinson's disease. Brain Research, 904(1), 93-103. https://doi.org/10.1016/S0006-8993(01)02489-1

Tseng, K. Y., Kasanetz, F., Kargieman, L., Riquelme, L. A., \& Murer, M. G. (2001). Cortical Slow Oscillatory Activity Is Reflected in the Membrane Potential and Spike Trains of Striatal Neurons in Rats with Chronic Nigrostriatal Lesions. Journal of Neuroscience, 21(16), 6430-6439.

Walters, J. R., Hu, D., Itoga, C. A., Parr-Brownlie, L. C., \& Bergstrom, D. A. (2007). Phase relationships support a role for coordinated activity in the indirect pathway in organizing slow oscillations in basal ganglia output after loss of dopamine. Neuroscience, 144(2), 762-776. https://doi.org/10.1016/j.neuroscience.2006.10.006

Wang, M., Wong, A. H., \& Liu, F. (2012). Interactions between NMDA and dopamine receptors: A potential therapeutic target. Brain Research, 1476, 154-163. https://doi.org/10.1016/j.brainres.2012.03.029

Wei, W., Rubin, J. E., \& Wang, X.-J. (2015). Role of the Indirect Pathway of the Basal Ganglia in Perceptual Decision Making. Journal of Neuroscience, 35(9), 4052-4064. https://doi.org/10.1523/JNEUROSCI.3611-14.2015

Weinberger, M., Mahant, N., Hutchison, W. D., Lozano, A. M., Moro, E., Hodaie, M., Lang, A. E., \& Dostrovsky, J. O. (2006). Beta Oscillatory Activity in the Subthalamic Nucleus and Its Relation to Dopaminergic Response in Parkinson's Disease. Journal of Neurophysiology, 96(6), 3248-3256. https://doi.org/10.1152/jn.00697.2006

Willard, A. M., Isett, B. R., Whalen, T. C., Mastro, K. J., Ki, C. S., Mao, X., \& Gittis, A. H. (2019). State transitions in the substantia nigra reticulata predict the onset of motor deficits in models of progressive dopamine depletion in mice. ELife, 8, e42746. https://doi.org/10.7554/eLife.42746 
bioRxiv preprint doi: https://doi.org/10.1101/2020.02.09.941161; this version posted February 10,2020 . The copyright holder for this preprint

(which was not certified by peer review) is the author/funder, who has granted bioRxiv a license to display the preprint in perpetuity. It is made available under aCC-BY 4.0 International license.

887 Zhuang, P., Hallett, M., Meng, D., Zhang, Y., \& Li, Y. (2019). Characteristics of oscillatory activity in the globus pallidus internus in patients with Parkinson's disease (P1.8-028). Neurology, 92(15 Supplement), P1.8-028.

890 
Figure 1, Whalen et al.
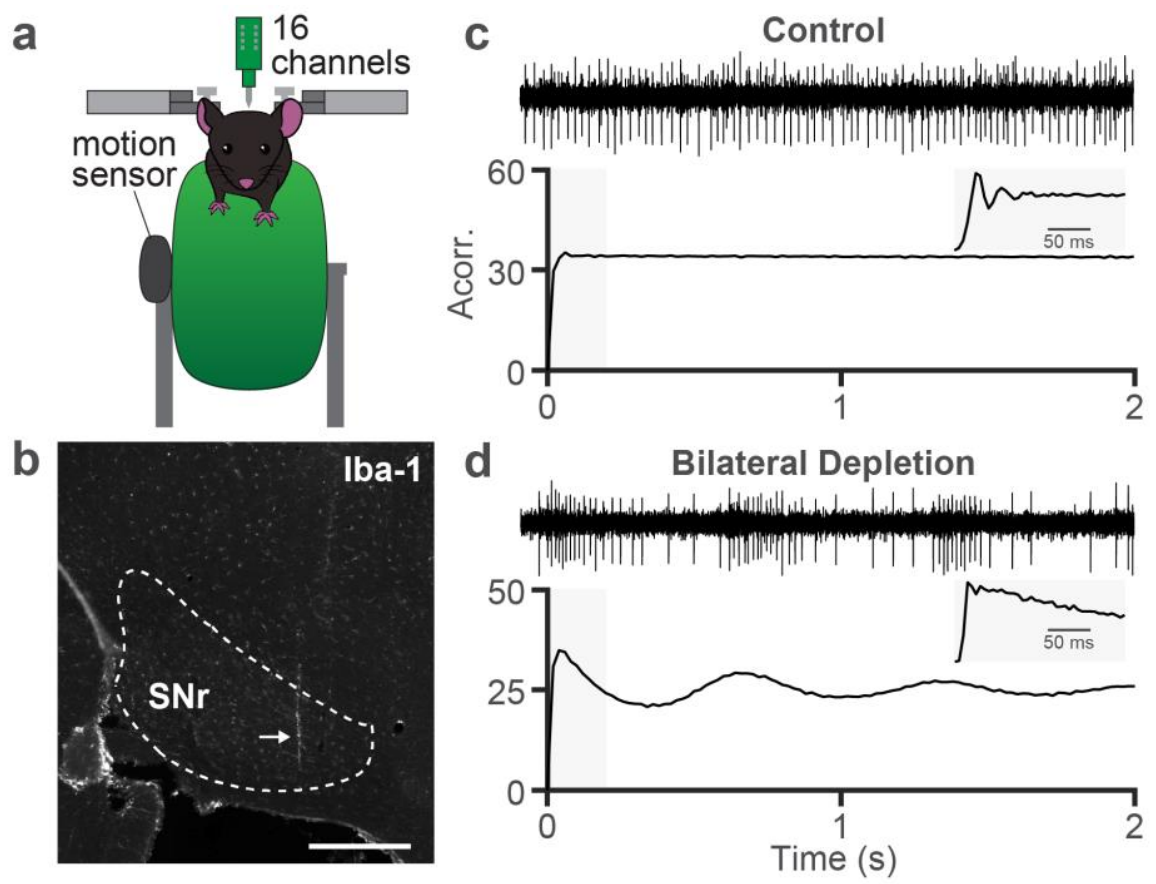

Figure 1. Dopamine depletion leads to low frequency spiking oscillations in SNr units.

a. Schematic of recording setup. Mice were head-fixed atop a free-running wheel with attached movement sensor and single units were recorded with a 16-channel probe. b. Example sagittal

894 slice with IBA immunofluorescence showing location of the recording probe in SNr. Dotted line 895 indicates approximate location of target nucleus, arrow indicates probe location. Scale bar = $896500 \mu \mathrm{m}$. c. Two seconds of an example SNr unit firing from a control animal (top) and the unit's 897 autocorrelation (bottom). Inset is zoomed into the first 200 milliseconds of the autocorrelation 898 using a smaller bin size. d. Same as c for a bilaterally dopamine depleted animal. 
Figure 2, Whalen et al.

\section{a}
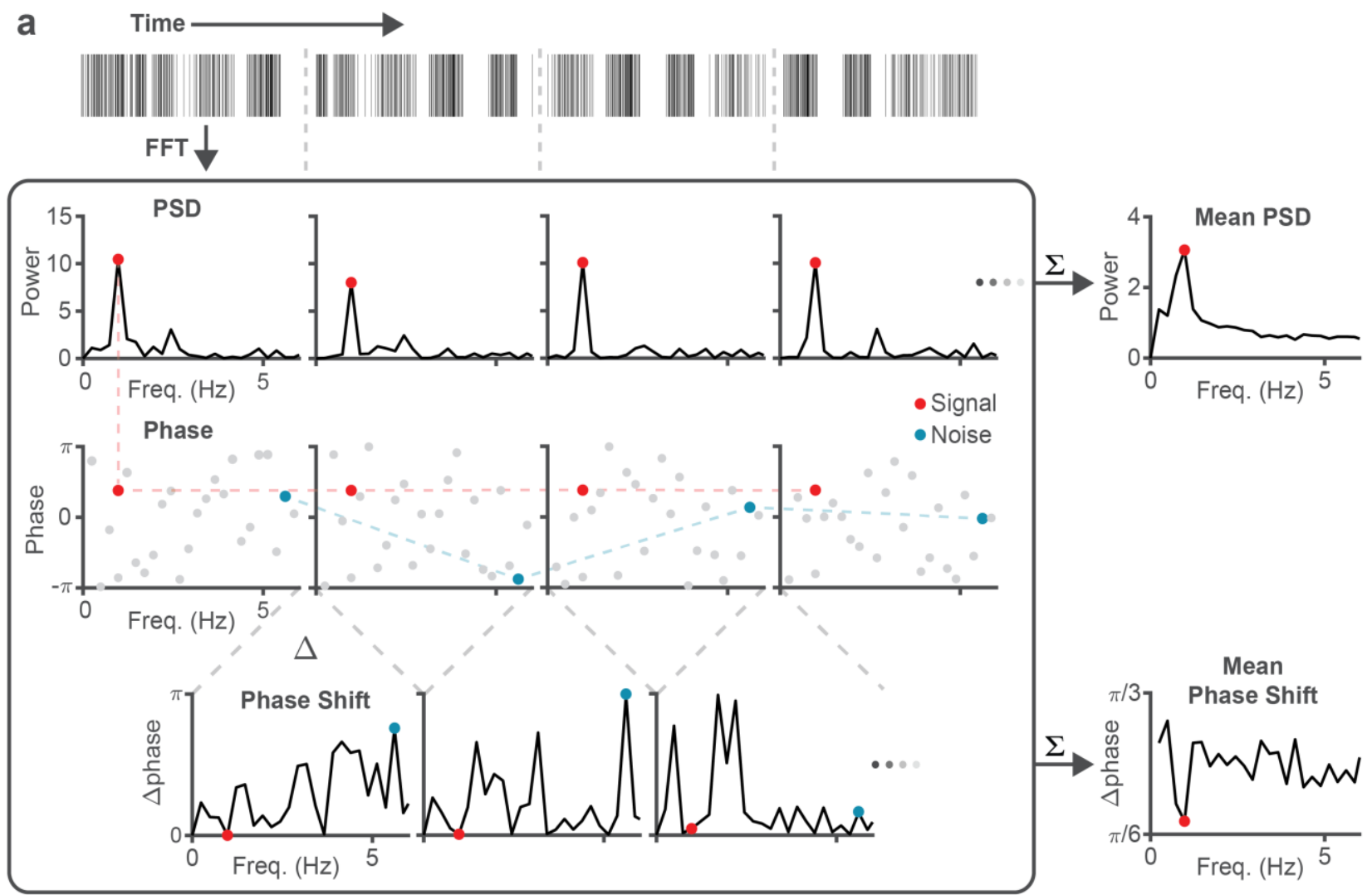

b
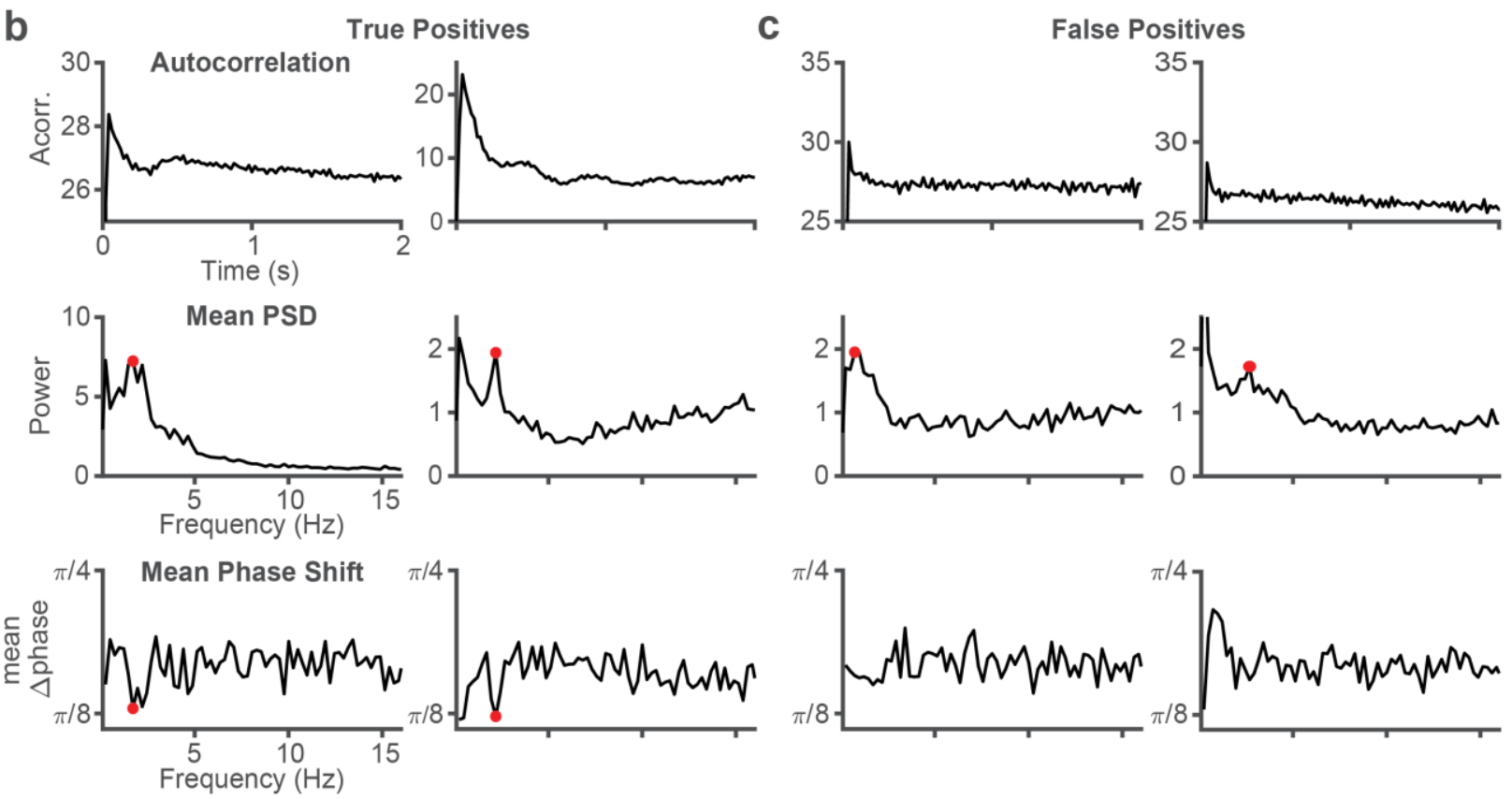

Figure 2. A phase shift measure to distinguish oscillations from noise.

900 a. Diagram of the phase shift oscillation detection method. A spike train is divided into

901 overlapping windows (1st row) and its Fourier transform is computed (corrected for the 
Figure 2, Whalen et al.

902 interspike interval distribution, see Methods). We identify statistically significant peaks in the 0.5-

$9033 \mathrm{~Hz}$ range (compared to a control $100-500 \mathrm{~Hz}$ range) in the averaged power spectral density

904 (PSD) across all windows (2nd row) and label the oscillation phase (3rd row) at that frequency.

905 Notice while the peak frequency (red) has consistent phase across windows, an arbitrary noise

906 frequency (blue) has inconsistent phase. We take the absolute circular difference of phases at

907 each frequency (4th row) and compute whether the frequency identified in the power spectrum

908 also has statistically significantly lower phase difference than the control band. A spike train

909 which has both a significant spectral peak and significant phase difference trough at the same

910 frequency is labeled as oscillating. b. Data from two example oscillating units. Top:

911 Autocorrelation exhibiting oscillations. Middle: Significant peaks (red dots) in the PSD

912 surrounded by pink noise. Bottom: The phase difference at these detected frequencies is

913 significantly lower than control frequencies. c. Same as $\mathbf{b}$, but for two units whose

914 autocorrelation appears to be non-oscillating yet have a peak in their PSD and which would be

915 "false positive" detections if only PSD's were analyzed without the consideration of phase shift. 
Figure 3, Whalen et al.
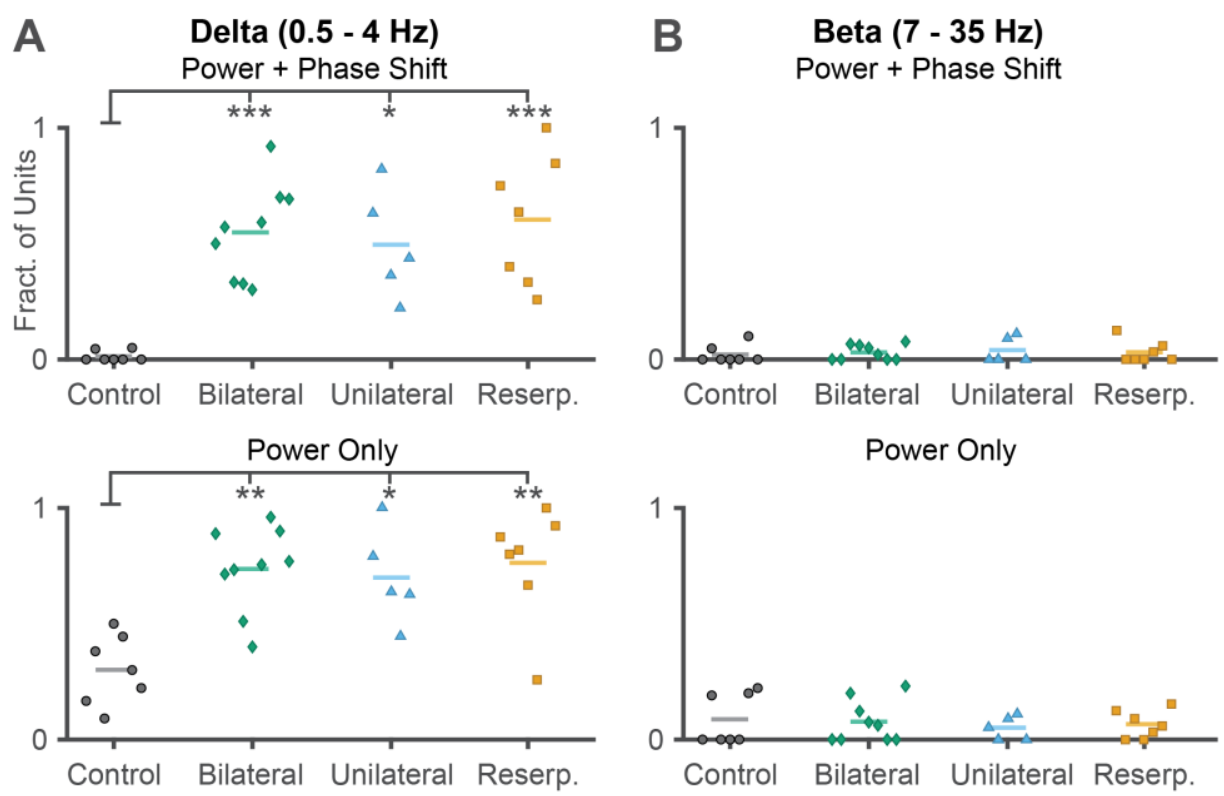

Figure 3. Dopamine depleted, but not control, SNr units exhibit phase-consistent delta

917 oscillations, but no change in beta oscillations.

918 Fraction of oscillating units from each animal in control conditions (black circles, $n=7$ ) or various methods of dopamine depletion - bilateral 6OHDA (green diamond, $n=9$ ), unilateral 6OHDA (blue triangle, $n=5$ ), or systemic reserpine (orange square, $n=7$ ). Lines indicate mean. a. Delta $(0.5-4 \mathrm{~Hz})$ oscillations detected using both PSD peak and low phase shift criteria. ANOVA: $p=5.206^{\star} 10^{-5} ;$ bilateral: $p=9.506^{\star} 10^{-5} ;$ unilateral: $p=0.00172$; reserpine: $p=$

$9235.908^{*} 10^{-5}$, Dunnett's post-hoc test. b. Same as a, but using only the spectral power criterion.

924 ANOVA: $p=4.668^{*} 10^{-4} ;$ bilateral: $p=5.645^{*} 10^{-4} ;$ unilateral: $p=0.00601$; reserpine: $p=$

$9255.794^{*} 10^{-4}$. c-d. Same as $\mathbf{a}-\mathbf{b}$, but for beta $(7-35 \mathrm{~Hz})$ oscillations. With phase shift, ANOVA: $p$

$926=0.8936$; without phase shift, ANOVA: $p=0.8908$ 
Figure 4, Whalen et al.

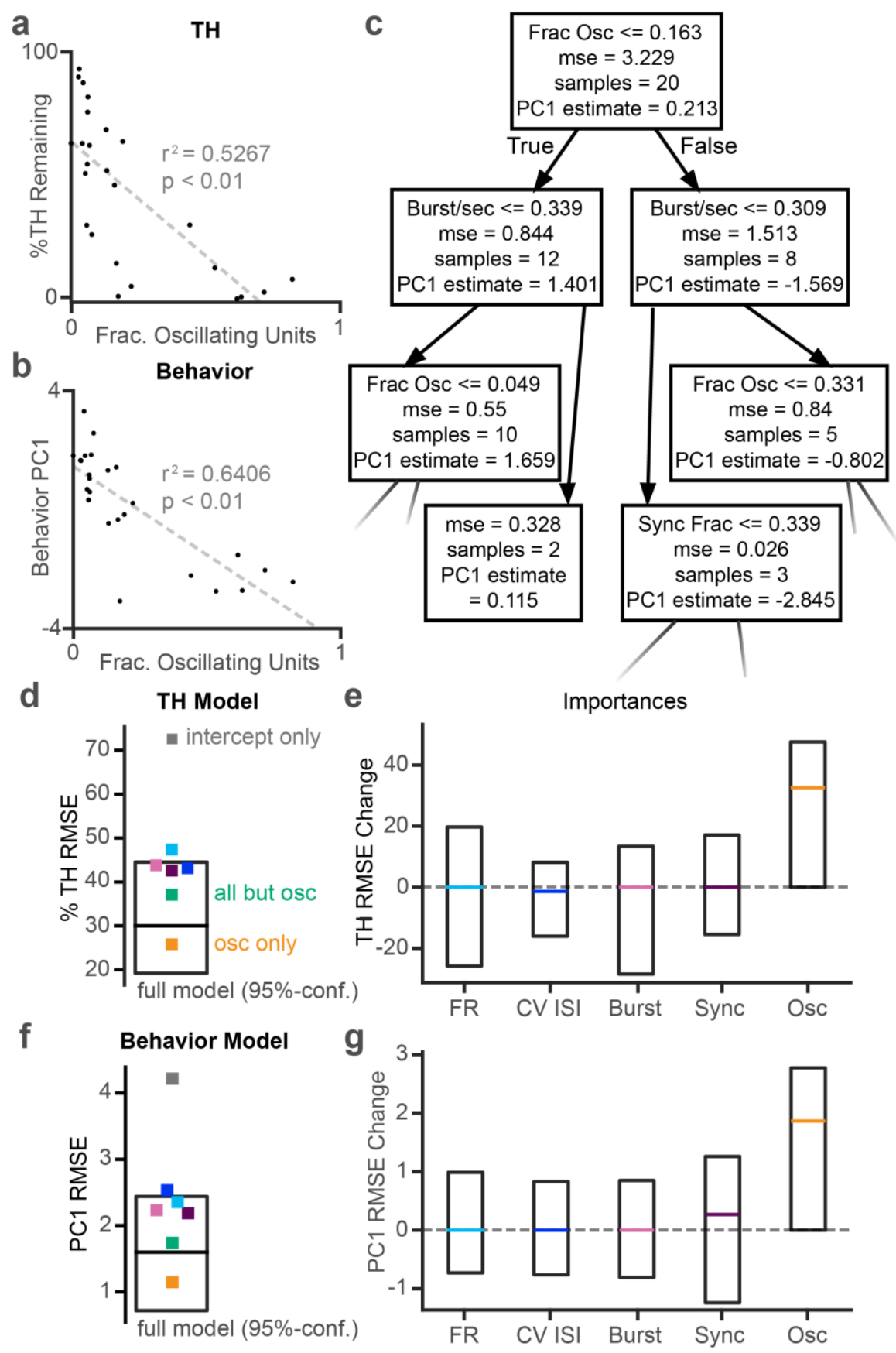

927 Figure 4. Oscillations predict severity of dopamine depletion.

928 a. Scatterplot showing relationship between levels of remaining striatal TH and fraction of

929 oscillating SNr units in animals $(n=25)$ gradually dopamine depleted to different severities. Each

930 dot denotes one animal, dashed line is the least squares fit. b. Same as a showing relationship

931 between the first principal component (PC1) of several behavioral metrics (see methods, more 
Figure 4, Whalen et al.

932 negative indicates more dysfunctional) and the fraction of oscillating SNr units. c. The first three

933 rows of one example decision tree predicting striatal $\mathrm{TH}$ from $\mathrm{SNr}$ neural properties (firing rate,

934 irregularity, burstiness, synchronicity and fraction of delta oscillating units). d. A 95\% confidence

935 interval of MSE from 1,000 trees predicting TH. Each square is the MSE of the median model

936 trained using a subset of parameters (grey: intercept-only, i.e. no parameters; light blue: firing

937 rate; dark blue: CV of interspike intervals; pink: bursts/second; purple: mean synchrony across

938 pairs; yellow: fraction of delta oscillating units; green: all parameters except fraction of delta

939 oscillating units). e. Middle 95 percentile (box) and median (colored line, same color scheme as

940 in d) of feature importances (permutation importance, see Methods) for each neural measure in

941 the TH model computed from 1,000 trees. Dotted line indicates zero importance. $\mathbf{f}-\mathbf{g}$. Same as

942 d-e for the model predicting PC1 of behavior. 
Figure 5, Whalen et al.

a

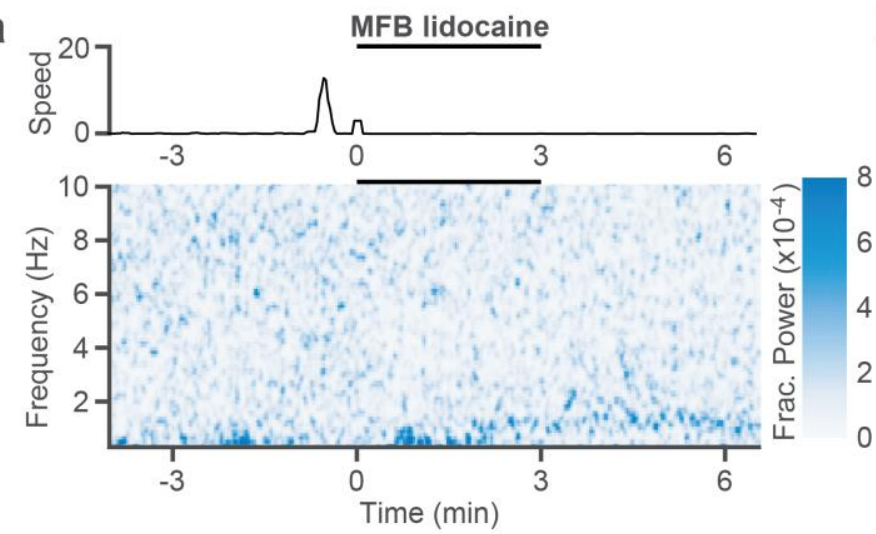

d
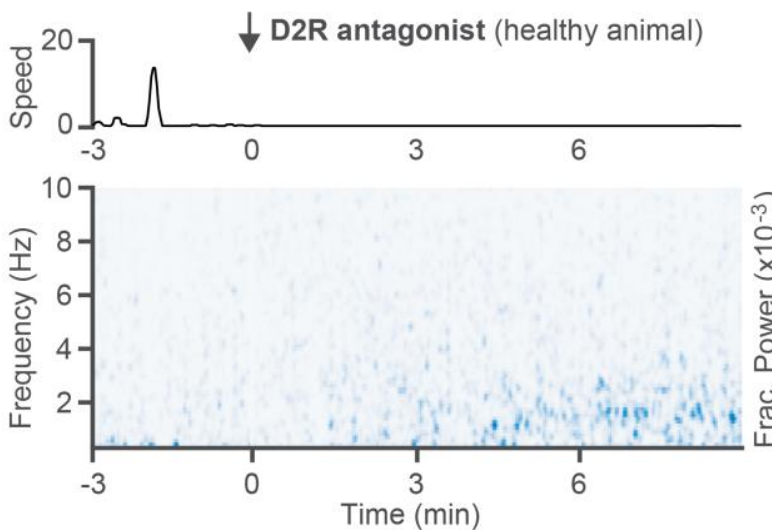

g
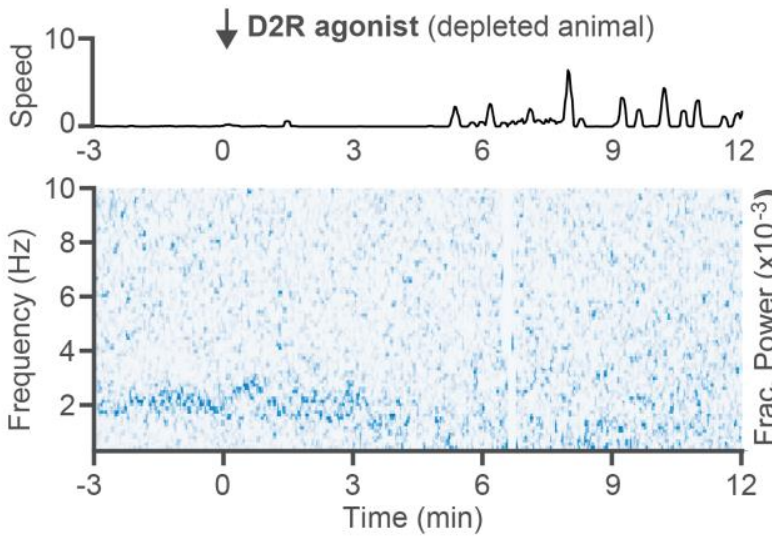

b
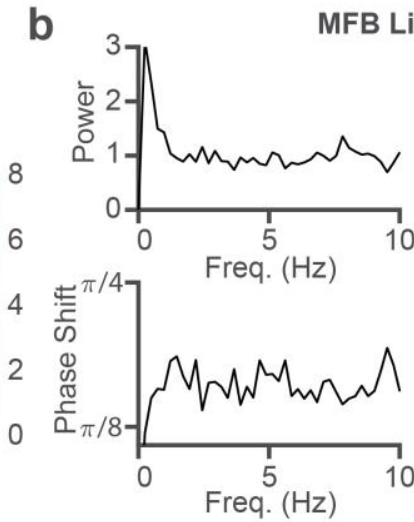

e
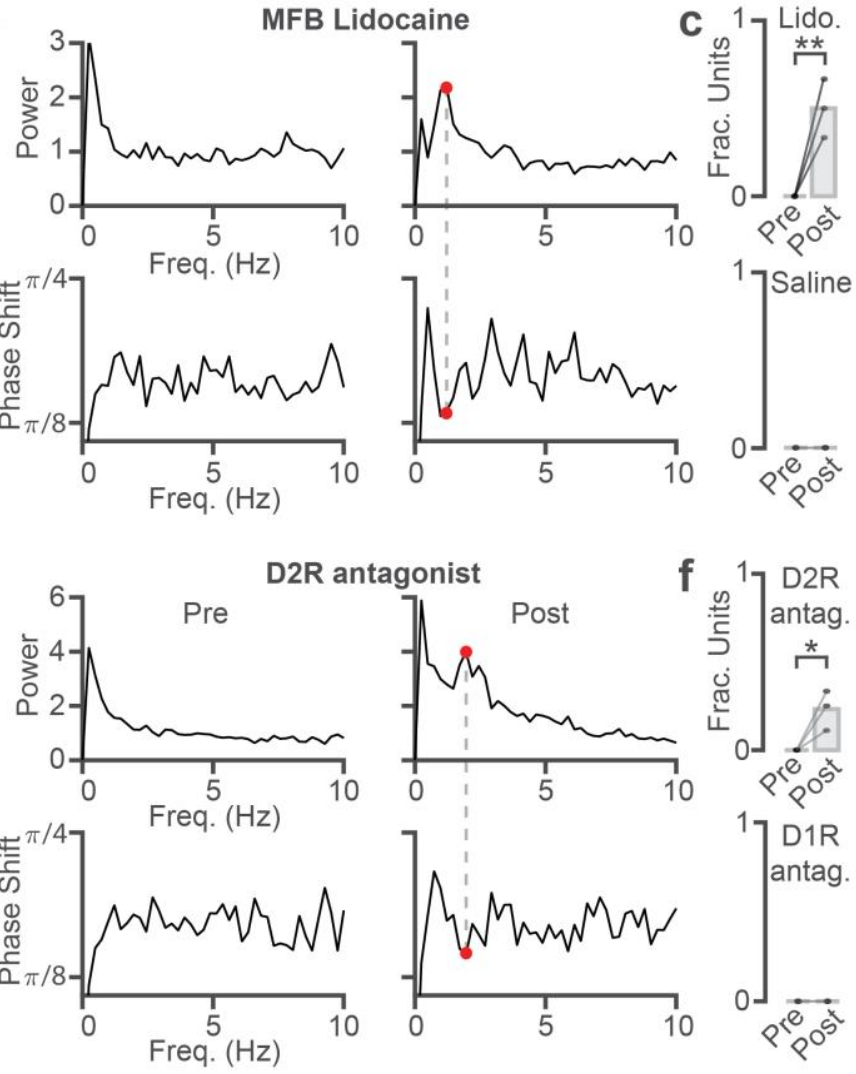

h

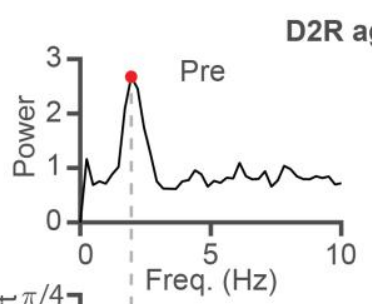

0.5
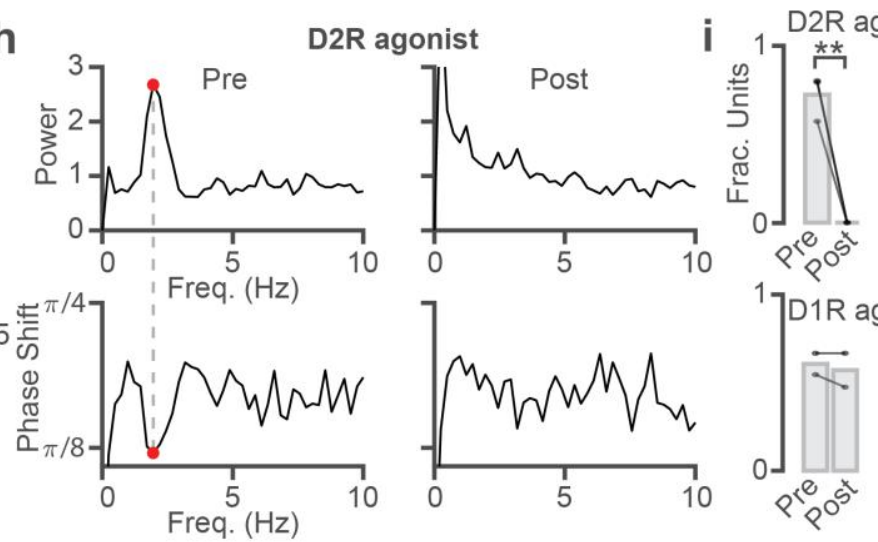

943

944

945

946 947 (right) lidocaine infusion. Bottom: Phase shift plots corresponding to the above PSDs. A dashed

948

Figure 5: Acute manipulations of MFB signaling or D2-receptors modulate oscillations.

a. Effects of lidocaine infusion into the MFB of healthy mice. Top: Speed of mouse on running wheel during lidocaine infusion (black bar). Bottom: Spike spectrogram of an example SNr unit during the same infusion as above. b. Top: PSDs from the same unit before (left) and after line from the detected oscillation in the right PSD (red dot) connects to the same frequency in 
Figure 5, Whalen et al.

949 the corresponding phase shift plot c. Fraction of oscillating units from all animals before and

950 after lidocaine (top, $n=3, p=0.00219$ ) or saline (bottom, $n=2, p=1.000$ ) infusion into the

951 MFB. Each dot is one animal, bars indicate mean, and lines connect the same animal before

952 and after infusion. d-f. Same as a-c, but for systemic injection of a D2R antagonist (raclopride,

$953 n=3, p=0.0233)$ compared to a D1R antagonist $(S C H 233890, n=2, p=1.000) \mathbf{g}-\mathbf{i}$. Same as

$954 \mathbf{d}-\mathbf{f}$, but for systemic injection of a D2R agonist (quinpirole, $n=3, p=8.686^{\star} 10^{-4}$ ) compared to a

955 D1R agonist (SKF81297, $n=2, p=0.7455)$ in dopamine depleted animals. 
Figure 6, Whalen et al.
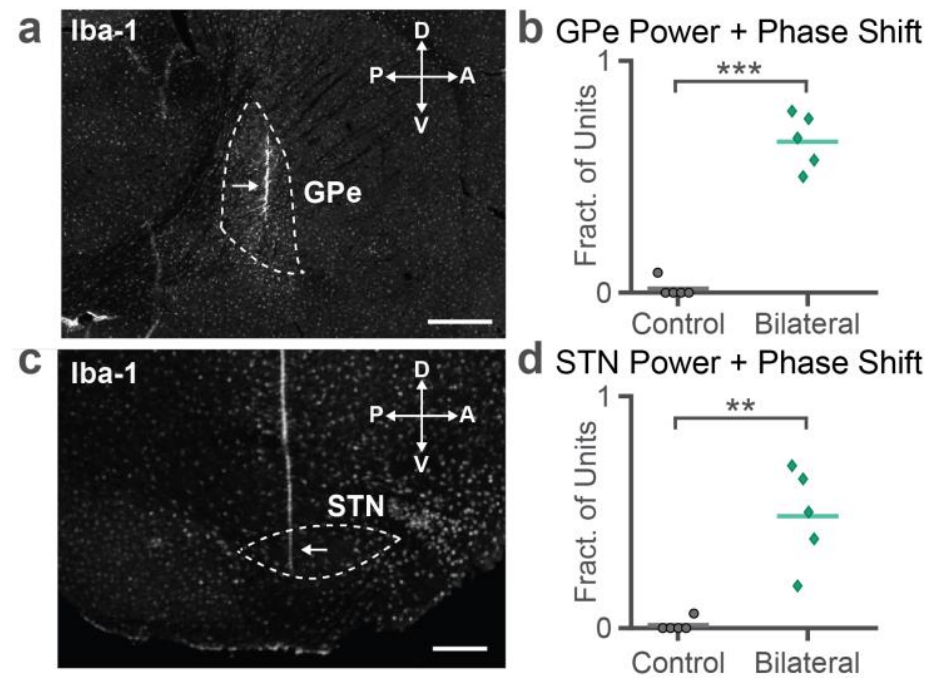

d STN Power + Phase Shift

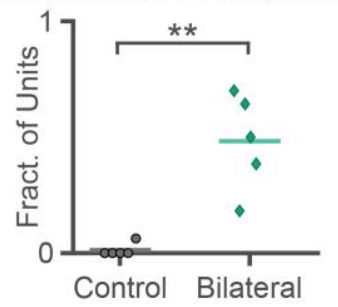

956 Figure 6: Delta oscillations pervade the dopamine depleted, but not healthy, indirect

957 pathway.

958 a. IBA immunofluorescence showing example probe locations in GPe. Dotted line indicates

959 approximate location of GPe, arrow indicates probe location. Scale bar $=500 \mu \mathrm{m}$. b. Fraction of oscillating units from each animal in control (black circles, $n=5$ ), or bilateral 6OHDA (green

961 diamond, $n=5)$ animals in GPe $\left(p=3 \cdot 847^{*} 10^{-6}\right.$, two-sample t-test $)$. $\mathbf{c}-\mathbf{d}$. Same as a-b targeting STN $(p=0.00106$, both control and bilateral $n=5)$ 
Figure 7, Whalen et al.

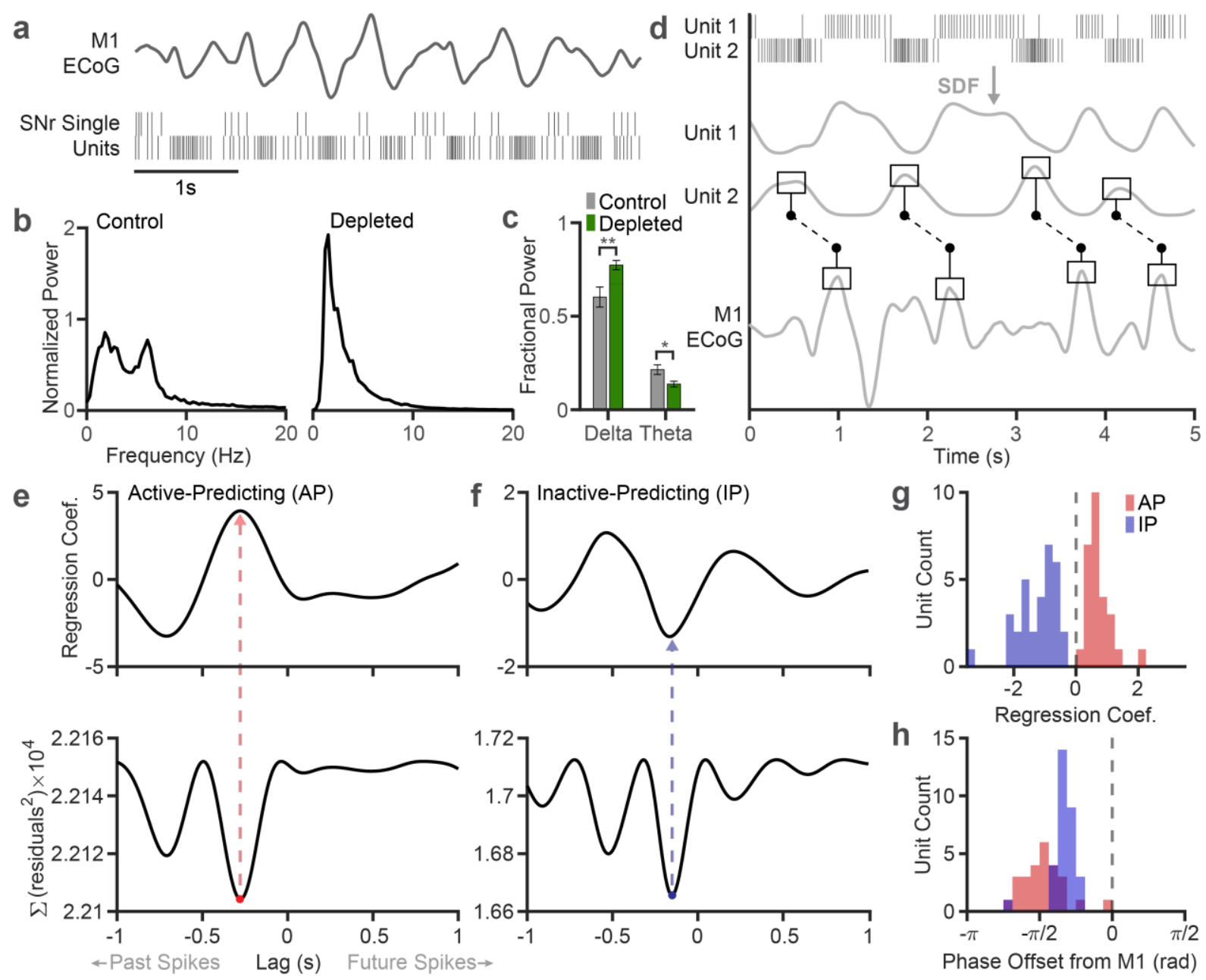

a. Example simultaneous M1 ECoG and spike trains from two SNr units exhibiting coherent oscillations. b. Example M1 ECoG power spectra from control (left) and bilaterally depleted

966 (right) animals. Power spectra were normalized to their total $0.5-100 \mathrm{~Hz}$ power and multiplied by

9671000 for visualization. c. Fractional delta and theta band power in M1 ECoG across all control (n $968=8)$ and acutely depleted $(n=9)$ animals. Bars indicate mean, error bars indicate standard error $969(p=0.00818$ for delta, 0.0173 for theta, two-sample t-test test). d. Example data demonstrating SNr predicting M1. Top: 5 second rasters from two simultaneously recorded SNr units. Middle:

971 spike density functions (SDF) of the above SNr rasters. Bottom: Simultaneously recorded M1 ECoG. Lines between the bottom two panels illustrate M1 exhibiting peaks at a consistent time 
Figure 7, Whalen et al.

973 lag after the peak of an SNr SDF, even amidst variance in oscillation period length. e. Example

974 regression results predicting M1 ECoG from an "active-predicting" (AP) SNr unit. Top:

975 Regression coefficients for each individual lag. Negative lag corresponds to SNr oscillations

976 leading M1. Bottom: MSE of regression results using each lag. The red dot indicates that the

977 model using that lag significantly outperforms an autoregressive model of the ECoG (F-test, $p<$

9780.05 correcting for multiple lag comparisons). The dotted line to the upper panel lands at a peak

979 in the coefficients, defining the unit as "active-predicting". f. Same as e for an "inactive-

980 predicting" (IP) SNr unit, whose significant lag is labeled in blue. g. Summary histogram of

981 regression coefficients from all oscillating SNr units recorded simultaneously with M1 ECoG ( $\mathrm{n}=$

982 59). Counts are colored as in e-f based on their regression coefficients (red: positive, blue:

983 negative, dashed line at zero), which define their type (AP or IP). $\mathbf{h}$. Same units colored as

984 above grouped by the phase offset at which they best predict the M1 ECoG (as in e-f, negative

985 phase offsets correspond to SNr oscillations leading changes in M1). 
Figure 8, Whalen et al.

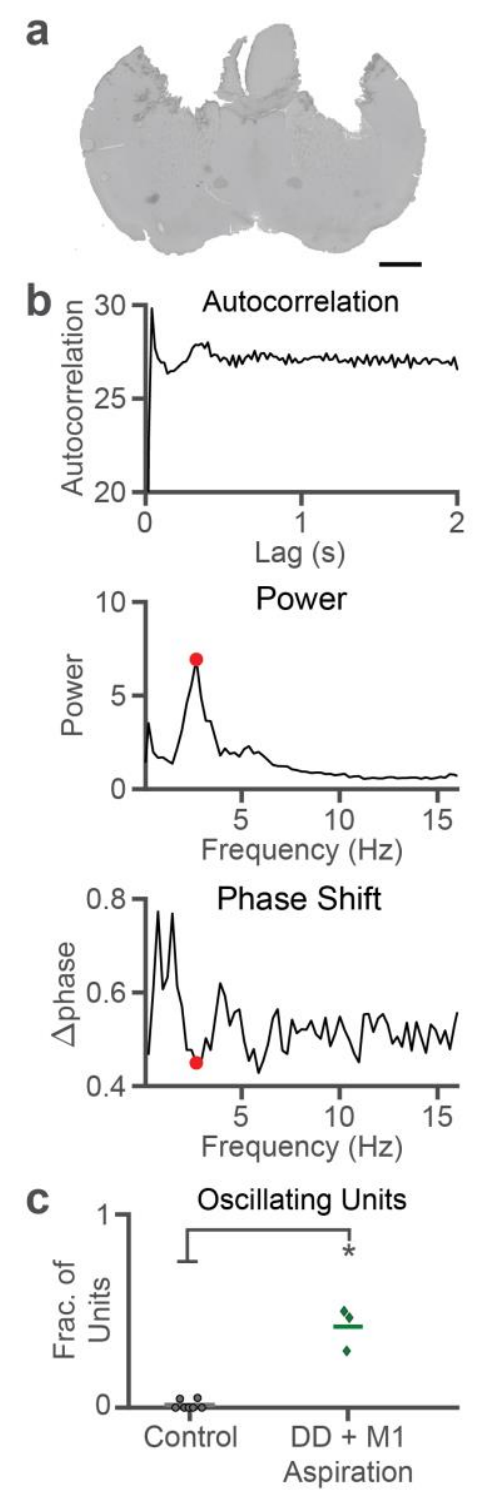

986 Figure 8: M1 lesion does not disrupt oscillations in SNr

a. Example coronal slice from an M1 lesioned animal. Scale bar $=1 \mathrm{~mm}$. b. Autocorrelation

988 (top), PSD (middle) and phase shift (bottom) for an example SNr unit exhibiting a delta oscillation in an M1-lesioned, dopamine depleted animal. c. Fraction of oscillating units in SNr

990 for each animal in control (black circle, $n=7$ ) and bilaterally dopamine depleted with M1 lesion

991 (dark green diamond, $n=3)$ conditions $\left(p=1.1478^{*} 10^{-6}\right.$, two-sample t-test.) 

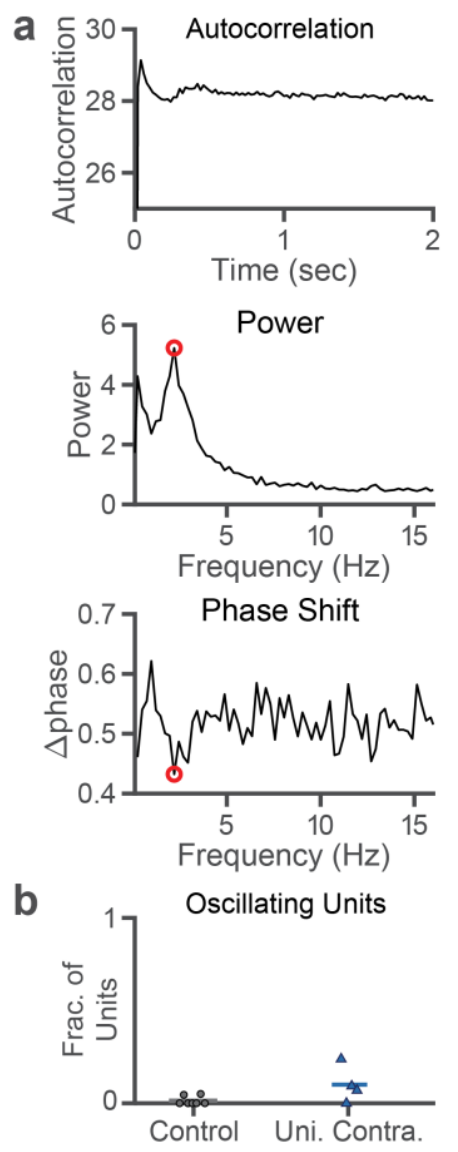

992 Figure 3 - Figure Supplement 1: Unilaterally depleted animals exhibit a small number of

993 delta oscillating units in the SNr of their dopamine intact hemisphere.

994 a. Example autocorrelation (top), PSD (middle) and phase shift (bottom) for an example SNr

995 unit exhibiting a delta oscillation in the intact hemisphere of a unilaterally depleted animal. b.

996 Fraction of oscillating units in SNr for each control animal (black circle, $n=7$ ) and in the intact

997 hemisphere of unilaterally depleted animals (dark blue triangle, $n=4$ ). The difference between

998 these conditions is not significant at the $\alpha=0.05$ level $(p=0.1138$, two-sample t-test). 


\section{Supplemental Figures, Whalen et al.}
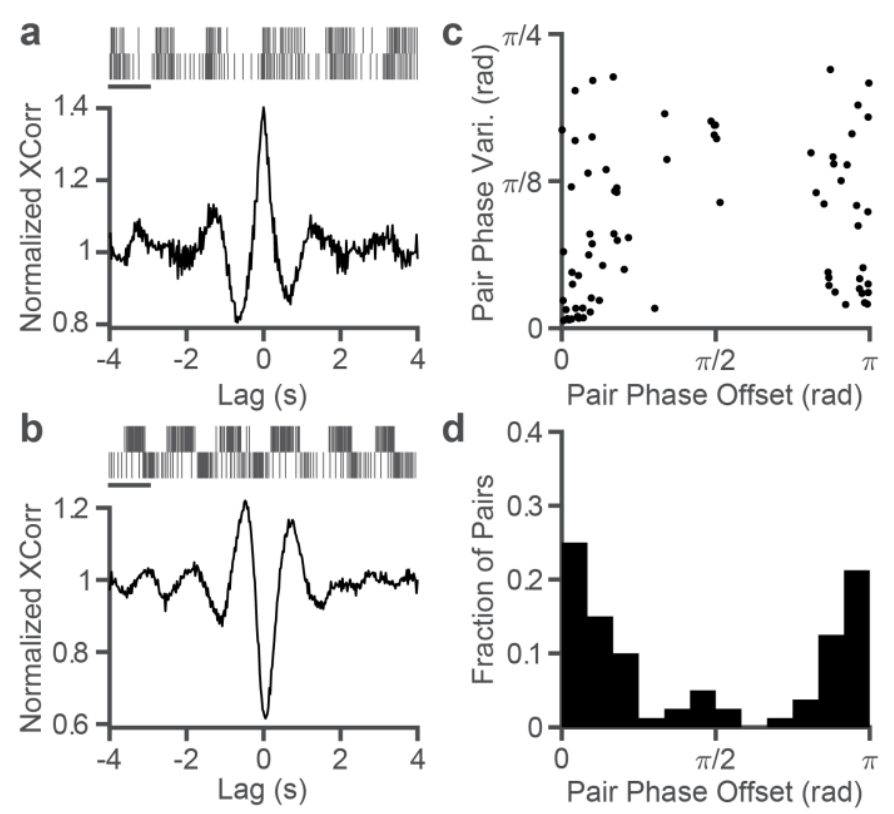

Figure 7 - Figure Supplement 1: Pairwise phase relationships corroborate the existence of two populations of oscillating units in dopamine depleted SNr.

a. Top: Spike rasters from a pair of simultaneously recorded SNr units, scale bar $=1 \mathrm{~s}$. Bottom:

1002 Normalized cross correlations (see Neural Measures section of Methods) of the above pairs

1003 demonstrating an in-phase relationship. b. Same as a for a near anti-phase relationship. c.

1004 Scatterplot of all pairs of oscillating units. The horizontal axis measures their mean phase offset 1005 ( 0 indicating in phase, $\pi$ indicating antiphase), and the vertical axis measures circular variance 1006 of phase offset computed across time windows. d. Histogram collapsing the above scatterplot to 1007 show counts of pairs based on their phase difference. 